OPEN ACCESS

Edited by:

Chang-Zhi Wu,

Chang'an University, China

Reviewed by:

Wei Zhang,

China University of Geosciences

Wuhan, China

Saeed Alirezaei,

Shahid Beheshti University, Iran

*Correspondence:

Qigui Mao

qg_mao@sina.cn

Specialty section: This article was submitted to

Economic Geology,

a section of the journal

Frontiers in Earth Science

Received: 31 December 2020

Accepted: 10 May 2021

Published: 16 June 2021

Citation:

Tan W, Mao Q, Yu M, Sun Y and Lv X (2021) Mineralization of the Tuwu

Porphyry Cu Deposit in Eastern

Tianshan, NW China: Insights From In

Situ Trace Elements of Chlorite

and Pyrite.

Front. Earth Sci. 9:648177.

doi: 10.3389/feart.2021.648177

\section{Mineralization of the Tuwu Porphyry Cu Deposit in Eastern Tianshan, NW China: Insights From In Situ Trace Elements of Chlorite and Pyrite}

\author{
Wei Tan ${ }^{1,2}$, Qigui Mao ${ }^{1 *}$, Mingjie $Y u^{1}$, Yan Sun ${ }^{1}$ and Xiaoqiang $L v^{1,2}$ \\ ${ }^{1}$ Beijing Institute of Geology for Mineral Resources Co., Ltd., Beijing, China, ${ }^{2}$ Sino-Zijin Resources Ltd., Beijing, China
}

The Tuwu porphyry copper deposit is located on the Dananhu-Haerlik island arc in eastern Tianshan, NW China. Based on geology, petrology, and in situ trace element studies of pyrite and chlorite, we redefined the characteristics of hydrothermal fluids and the following three mineralization stages: premineralization stage (stage I), porphyry metallogenic stage (stage II), and superimposed transformation stage (stage III). Pyrite stage I (Py-I) has the highest $\mathrm{Co} / \mathrm{Ni}$ ratios, and the precipitation crystallization of chlorite $\left(\mathrm{Chl}-\mathrm{I}_{2}\right)$ has the similar rare earth element distribution patterns with those of volcanic rocks Carboniferous Qieshan (CQ), indicating intense volcanic hydrothermal activity. The Co/Ni ratios of Py$\|_{1}$ and Py- $\|_{2}$ (stage II) tend to decrease over time. Moreover, the rare earth element (REE) distribution patterns of Chl-II have similar LREE enrichment, and the Eu anomalies in Chl$\left\|_{1}, \mathrm{Chl}-\right\|_{2}$, and Chl- $\|_{3}$ range from positive to negative. The initial ore-forming fluid was mainly magmatic hydrothermal fluid, and with the late-stage addition of meteoric water and continuous sulfide precipitation, the trace element composition of the fluid changed, and the whole system became more oxidizing. Py-III (stage III) has the lowest Co/Ni ratios, and the REE distribution pattern of Chl-III is characterized by LREE enrichment. Moreover, the Chl-IIl shows obvious shear deformation characteristics. The results indicate that the host rocks experienced intensely superimposed reformation. By combining and integrating our results with the regional evolution processes in the eastern Tianshan, we propose that the Tuwu porphyry deposit has undergone magmatic hydrothermal and metamorphic hydrothermal processes. Volcanism (stage I) provided the space and initial conditions for the emplacement of the metallogenic body. With the emplacement of the plagiogranite porphyry (stage II), the main copper mineralization occurred in the porphyry and surrounding rocks. After porphyry mineralization (stage III), regional ductile shearing and collisional compression led to a copper reaction, and its accumulation along the faults formed an ore shoot.

Keywords: porphyry deposits, mineralization, pyrite, chlorite, LA-ICP-MS 


\section{INTRODUCTION}

The eastern Tianshan orogen, which represents an important part of the tectonic evolution of the Central Asian orogenic belt, has undergone a complicated evolutionary process of stretching, multiblock merging, subduction accretion, collisional orogenesis, and postcollision processes (Figure 1A) (Chen et al., 2007; 2012; Pirajno, 2009, 2013; Zhai et al., 2011). Along with the multiple processes of continental accretion, crust-mantle interactions, and transformation in the eastern Tianshan orogen, numerous polygenetic mineral deposits have been formed, and most have been superimposed by late complex ore-forming processes. These deposits include the Honghai (Huangtupo) $\mathrm{Cu}-\mathrm{Zn}$ deposit (Deng et al., 2016; Mao et al., 2019), Xiaorequanzi $\mathrm{Cu}-\mathrm{Zn}$ deposit (Long et al., 2019; Mao et al., 2020), Yamaisu Cu-Fe deposit, Hongyuntan Cu-Fe deposit (Sun et al., 2017; Han et al., 2018; Zhang et al., 2018), and Tuwu-Yandong $\mathrm{Cu}$ deposit (Wang Y. F. et al., 2016; Xiao et al., 2017). Research on superimposed metallogenic systems is very important for recognizing the regional metallogenic history and guiding mineral exploration activities in the eastern Tianshan.

The Tuwu porphyry copper deposit is located at the southern part of the Dananhu-Haerlik island arc belt (Figure 1B). Since the Tuwu copper deposit was discovered by the No. 1 geological party of the Xinjiang Bureau of Geological Exploration in 1994, the Yandong, Fuxing, Yanxi, Linglong, and Chihu copper deposits were consecutively discovered afterward. All of these deposits formed the Tuwu-Yandong porphyry copper metallogenic belt, which has attracted extensive attention. Studies have focused on its geological characteristics (Wang et al., 2001; Rui et al., 2001, 2002; Han et al., 2006; Shen et al., 2014b), metallogenic background, metallogenic characteristics, zoning classification (Shen et al., 2012; Pan et al., 2013; Xiao et al., 2018), geochemistry, geochronology (Rui et al., 2002; Zhang et al., 2006; Han et al., 2014; Shen et al., 2014a; Wang et al., 2014), metallogenic chronology (Rui et al., 2002; Wang et al., 2014; Xiao et al., 2017), and compositions and characteristics of oreforming fluids (Han et al., 2006; Gao et al., 2015; Wang et al., 2015, 2017). We have found that there are many differences between the porphyry metallogenic system of the Tuwu-Yandong deposit and the classical porphyry metallogenic model. For example, 1) the boundaries of the potassic zone are not obvious, 2) the morphology of the ore body is tabular rather than equiaxial, and 3) the temperature of the ore-forming fluid is obviously lower than that of a typical porphyry copper deposit. These findings indicate that the Cenozoic porphyry mineralization model may not be strictly suitable for Paleozoic porphyry deposits which experienced a multistage structural evolution.

Mineral microanalysis is an important method for defining ore-forming fluid characteristics, metallogenic physicochemical conditions, and ore-forming element migration and enrichment processes (Large et al., 2009; Zhou, et al., 2010; Ye et al., 2011; Zhang, 2011; Reich et al., 2013; Franchini et al., 2015; Zhang et al., 2020). The development of laser ablation inductively coupled plasma mass spectrometry (LA-ICP-MS) analysis technology has allowed for the measurement of most trace elements in sulfide and oxides. Pyrite is an important sulfide in many metallogenic systems, and its chemical composition is often used to trace the genesis of ore deposits and the metallogenic environment and conditions (Cook et al., 2009; Large et al., 2009, 2011; Reich et al., 2013; Franchini et al., 2015). Chlorite is a mineral that commonly forms during diagenesis, medium- to lowtemperature metamorphism and hydrothermal alterations. Due to the variability in its structure and the nonstoichiometry of its composition, chlorite and its paragenetic mineral assemblages are often used to constrain the physicochemical conditions and determine the diagenetic and metallogenic environment (Davidson et al., 2001; Bortnikov et al., 2008; Inoue et al., 2010; Fadda et al., 2012; Wilkinson et al., 2015; David et al., 2016). This study used LA-ICP-MS to analyze the composition of trace elements in pyrite and chlorite in the main alteration zone of the Tuwu copper deposit, and the combination of detailed geological surveys and petrographical studies was used to study the genesis of ore minerals and the characteristics of trace elements and further reveal characteristics of hydrothermal fluids and mineralization processes of the Tuwu porphyry deposit.

\section{REGIONAL GEOLOGICAL BACKGROUND}

A review of the regional geology and the tectonic evolution processes of the accretionary orogenic belt from the Precambrian to Triassic are given in Xiao et al. (2004). From north to south, eastern Tianshan consists of the Dananhu-Haerlik arc, Kangguer Forearc, Yamansu arc, Central Tianshan arc, and the South Tianshan (Figure 1B).

The Dananhu-Haerlik Ordovician-Carboniferous island arc, as an important Ordovician to Permian metallogenic belt located between the Kalameili and the Kangguer faults (Figure 1B), includes the Paleozoic Dananhu-Haerlik arc, the Bogda Late Paleozoic intra-arc basin, and the Kanggur forearc (Xiao et al., 2004, 2015). The Dananhu-Haerlik arc consists of Ordovician to Permian tholeiite to calc-alkaline mafic-felsic volcanic rock, volcanoclastics, tuff, and flysch sediments (Ma et al., 1997; Li et al., 2004; Xiao et al., 2004; Hou et al., 2005; Tang et al., 2006; Mao et al., 2014b, Mao et al., 2016; Deng et al., 2016; Li et al., 2016). The Ordovician to Silurian marine volcanic and volcaniclastic rocks consist of tholeiite to calc-alkaline basalts, andesite-basalts, andesite, high-Mg andesite, and volcaniclastic rocks (Ma et al., 1997; Mao et al., 2014b, 2016; Deng et al., 2016; Li et al., 2016). The Devonian to Carboniferous rocks mainly consists of tholeiite to calc-alkaline basalts, andesite, dacite rhyolite, volcaniclastic rocks, and sedimentary (Ma et al., 1997; Li et al., 2004; Xiao et al., 2004; Hou et al., 2005; Tang et al., 2006; Mao et al., 2014b). The arc-related granitic intrusions are formed from Ordovician to Permian (Song et al., 2002; Li et al., 2004; Chen et al., 2005; Hou et al., 2005; Sun et al., 2005; Wu et al., 2006; Chao et al., 2006; Guo et al., 2006; Mao et al., 2010, 2019; Long et al., 2016; Zhang et al., 2017).

The Bogda intra-arc basin, which formed within the Dananhu-Haerlik arc from the Carboniferous to Early 

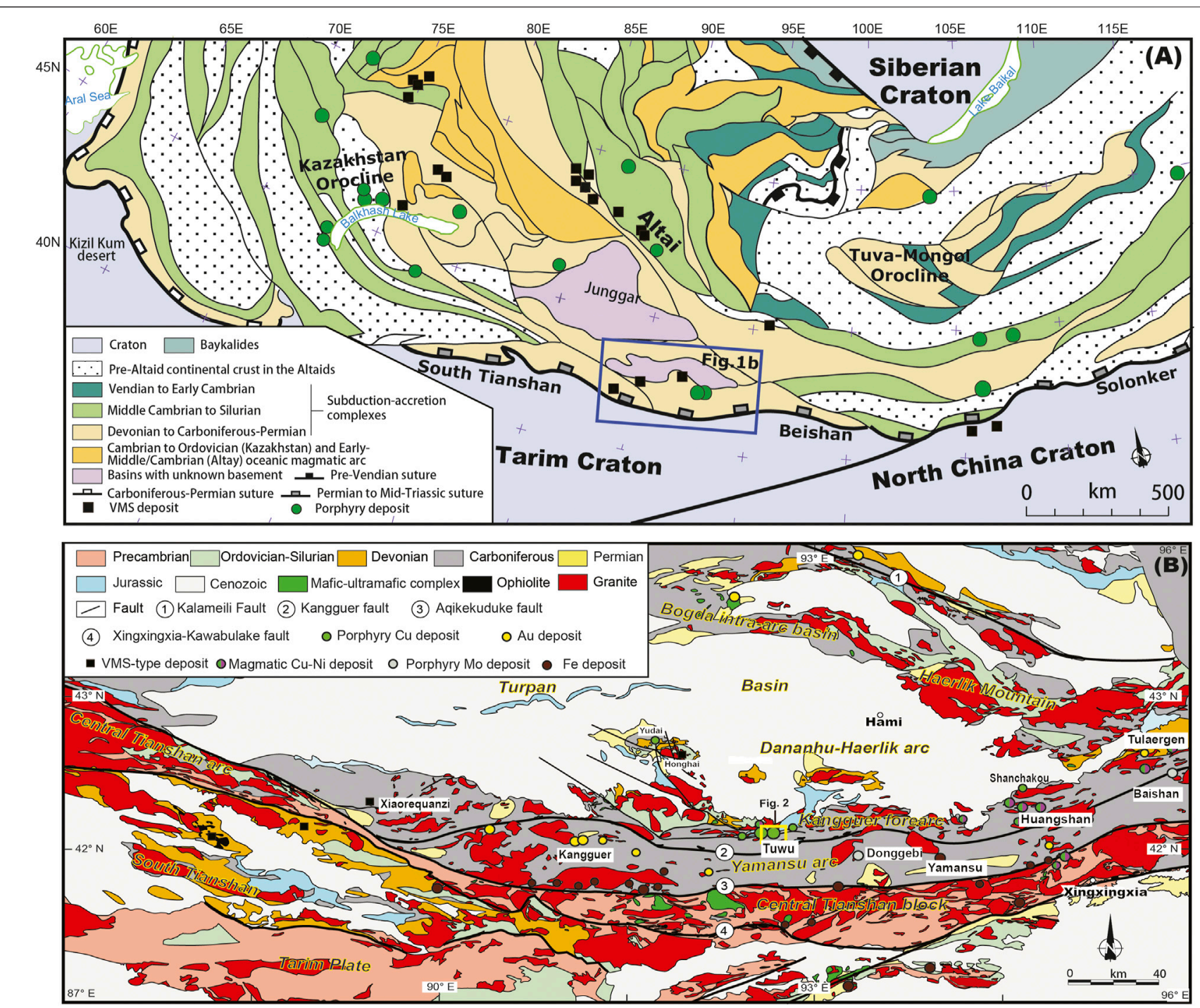

FIGURE 1 | (A) Schematic tectonic map of Central Asia (Şengör et al., 1993; Xiao et al., 2015) showing the position of the eastern Tianshan. (B) Schematic geological map of the eastern Tianshan [modified after Mao et al. (2017) and Xiao et al. (2004)] showing the location of the Tuwu area. The major faults separating the southern Tianshan, Central Tianshan arc, Yamansu arc, and Dananhu-Haerlik arc.

Permian, is composed of basalt, dacite, rhyolite, volcanoclastics, minor interbedded andesite, and sedimentary rocks. The mafic to granitic intrusions are mainly Early Permian (Gu et al., 2001a, Gu et al., 2001b; Li et al., 2004; Xiao et al., 2004; Li et al., 2006; Wang et al., 2010; Shu et al., 2011; Chen et al., 2013; Gao et al., 2014).

The Kanggur forearc located at the south margin of the Dananhu-Haerlik arc is made up of structurally dismembered Devonian-Carboniferous volcanic-sedimentary rocks and ophiolitic slices (Yang and Scott, 1996; Ma et al., 1997; Zhou et al., 2001; Xiao et al., 2004). The Permian granitoids are extensive, and a zone of earlier Permian mafic-ultramafic complexes is located along the Kangguer forearc terrane and stretches several hundred kilometers (Ma et al., 1997; Mao et al., 2002; Han C. M. et al., 2004; Xiao et al., 2004; Zhou et al., 2004; Qin et al., 2011). Multiple types of mineral deposits developed in the Dananhu-Haerlik arc, including porphyry $\mathrm{Cu}(\mathrm{Au} / \mathrm{Mo})$ deposits (Rui et al., 2002; Chen et al., 2005; Wu et al., 2006; Zhang et al., 2006; Shen et al., 2014a; Wang et al., 2015; Mao et al., 2018), hydrothermal vein $\mathrm{Cu}(\mathrm{Au})$ deposits (Fang et al., 2002; Mao et al., 2014a; Yu et al., 2019), Cu-Ni sulfide deposits (Mao et al., 2002; Han B. F. et al., 2004; Xiao et al., 2004; Zhou et al., 2004; Mao et al., 2006; Han et al., 2010; Qin et al., 2011), VMS Cu$\mathrm{Zn}-(\mathrm{Au} / \mathrm{Ag})$ polymetallic deposits (Mao et al., 2010, 2019; Deng et al., 2016; Li et al., 2002), and Au deposits (Wang et al., 2006).

The Dananhu-Haerlik island arc, situated north of the Kanggur fault, comprises Paleozoic volcano-sedimentary rocks and Mesozoic to Cenozoic sedimentary rocks, intercalated with volcanic and pyroclastic rocks (Xiao et al., 2004; Hou et al., 2006; Qin et al., 2011). Paleozoic mafic-ultramafic complexes and granites intruded the sedimentary and volcanic rocks and are 
associated with some $\mathrm{Cu}$ and $\mathrm{Au}$ mineralization (Deng et al., 2016; Gao et al., 2015). The Carboniferous rocks are mostly foliated and mylonitized and influenced by ductile shearing processes. Carboniferous acidic igneous rocks and a Permian mafic-ultramafic complex are well developed in this belt and mainly occur in the form of stocks (Han et al., 2006; Pan et al., 2013; Shen et al., 2014b). Late Paleozoic mineral deposits are developed at the southern zone of the Dananhu-Haerlik island arc. These deposits include shear zone-type gold deposits, such as the Tianmu Au deposit and Hongtan Au deposit (Yao, 2006), CuNi sulfide deposits, which are associated with Permian maficultramafic rocks (Wang Y. L. et al., 2016), and CarboniferousPermian porphyry $\mathrm{Cu}(\mathrm{Mo})$ deposits, such as the Tuwu-Yandong $\mathrm{Cu}$ deposits (Pirajno, 2013; Shen et al., 2014b) and Sanchakou Cu deposit (Qin et al., 2009).

\section{GEOLOGY OF THE TUWU DEPOSITS}

The Tuwu copper deposit is hosted in the Carboniferous Qieshan (CQ) group, diorite porphyrite and plagiogranite porphyry at the northeastern part of the Qieshan anticline (Figure 2A). According to previous studies (Han et al., 2006; Shen et al., 2012; Pan et al., 2013; Shen et al., 2014b), the CQ group can be divided into five lithologic members (Figure 2A). The first lithologic member $\left(\mathrm{CQ}^{1}\right)$ is volcanic effusive facies distributed symmetrically around the volcanic crater. The lithology is mainly basalt, followed by andesite, tuff, dacite, breccia lava, volcanic breccia, and other volcanics. Additionally, dioritic porphyrite, which is the main ore-hosting rock, is located at the contact between the basalt in $\mathrm{CQ}^{1}$ and the pebble-rich lithic sandstone in $\mathrm{CQ}^{3}$. The second lithologic member $\left(\mathrm{CQ}^{2}\right)$, which is an explosive volcanic facies, is located in the north-central part of the mining area near the center of volcanism. The lithology is agglomerate breccia lava and minor andesite and basalt. The second lithologic group can be divided into three lithologic members. The third lithologic member $\left(\mathrm{CQ}^{3}\right)$ is distributed in a nearly east-west direction in the northern part of the mining area. The lithology is mainly volcanic-sedimentary facies. $\mathrm{CQ}^{3}$ is a pebble-rich lithic sandstone that contains basalt and andesite layers. The fourth lithologic member $\left(\mathrm{CQ}^{4}\right)$ is distributed in the southern part of the mining area in a nearly east-west direction along the northern side of the Kangguer fault. The lithology is mainly pebble-rich sandstone and minor basalt and volcanic breccia at the top. The fifth lithologic member $\left(\mathrm{CQ}^{5}\right)$ is in angular unconformable contact with the underlying breccia lava of $\mathrm{CQ}^{2}$. The lithology is mainly heterolithic conglomerate with lenticular feldspathic lithic sandstone. The volcanic rocks of the CQ Group constitute a relatively complete volcanic eruption cycle and resulted from Carboniferous magmatism in this area. The CQ group strikes nearly east-west and dips to the south at angles of approximately $40-65^{\circ}$. The upper Carboniferous Gandun formation $\left(\mathrm{C}_{2} \mathrm{~g}\right)$ is distributed in the southern part of the mining area in a nearly east-west direction along the southern side of the Kangguer fault. The lithology is phyllonite, carbonaceous phyllonite and tuff, which have been altered by regional ductile shear deformation metamorphism. The Jurassic Xishanyao formation (Jx) is covered in cap rock and exposed in the northern part of the mining area. The lithology is mainly sandstone, siltstone, mudstone, and conglomerate.

The magmatism was developed quite intensely in the Tuwu-Yandong ore district. The intrusive rocks mainly include granite, plagiogranite porphyry, diorite and diorite porphyry. The Carboniferous metallogenic plagiogranite porphyry of the Tuwu deposit occurs as a small stock (Cheng et al., 2005; Wang et al., 2014; Xiao et al., 2018). The plagiogranite porphyry cut through the dioritic porphyrite and intrude into the contemporaneous volcanism-related fault with an E-W trend (Pan et al., 2013). The dioritic porphyrite is the main ore-hosting rock and belongs to subvolcanic rocks (Hou et al., 2005, 2006; Zhao et al., 2014). It cuts through the basalt in $\mathrm{CQ}^{1}$ and the pebblerich lithic sandstone in $\mathrm{CQ}^{3}$, thus forming an east-west-trending stock or dike (Shen et al., 2012) (Figure 3).

The Tuwu deposit is located in the northeastern part of the Qieshan anticlinorium (Figure 2A). The direction of fracture development is mainly east-west, followed by northwest-southeast. The copper ore bodies mainly occur in the dilated portion of the east-west fault. The intensity of the ductile deformation zone in the Tuwu-Yandong mining area is stronger in the south and weaker in the north. The southern part of the ore field features a continuous strong ductile zone, whereas the northern part is a weakly ductile deformation zone composed of several discretely distributed small lenticular and zonal ductile deformation zones. The copper ore body is distributed in an en echelon shape both in cross section and in map view. In general, the orecontrolling brittle-ductile shear zone is a ENE-trending tectonic belt that dips southward (Ma et al., 2003). The exploration data show that the schistosity obviously cuts the strata and ore body, indicating that the ore body suffered late brittle-ductile deformation. However, distinguishing radial and annular contemporaneous faults from the effect of brittle-ductile deformation is difficult.

The ore mainly has medium- to fine-grained hypidiomorphic-xenomorphic granular textures (Figure 4; Figure 5). The ore structure mainly includes veins and dissemination. The metallic minerals are mainly chalcopyrite, followed by pyrite and bornite, with minor chalcocite, covellite, magnetite, hematite, and molybdenite. Pyrite is mainly developed at the top and bottom of the ore body and it is negatively related to copper minerals. The gangue minerals are mainly quartz, sericite and chlorite, followed by biotite, epidote, actinolite, gypsum, feldspar, and calcite. The supergene minerals are malachite, atacamite, and limonite.

Propylitization is extensively developed in the volcanicpyroclastic rocks of the CQ Group. According to systematic alteration mapping and a comprehensive laboratory study, we find that weak propylitization related to volcanic hydrothermal activity in the late volcanic activity is concentrated in the range of $1.2-3 \mathrm{~km}$ from the ore body while strong propylitization obviously superimposed by late hydrothermal activity is concentrated in the range of $0-1.2 \mathrm{~km}$. The alteration intensity differs significantly between these zones, with the F2 fault as the dividing line. The altered minerals associated with weak 

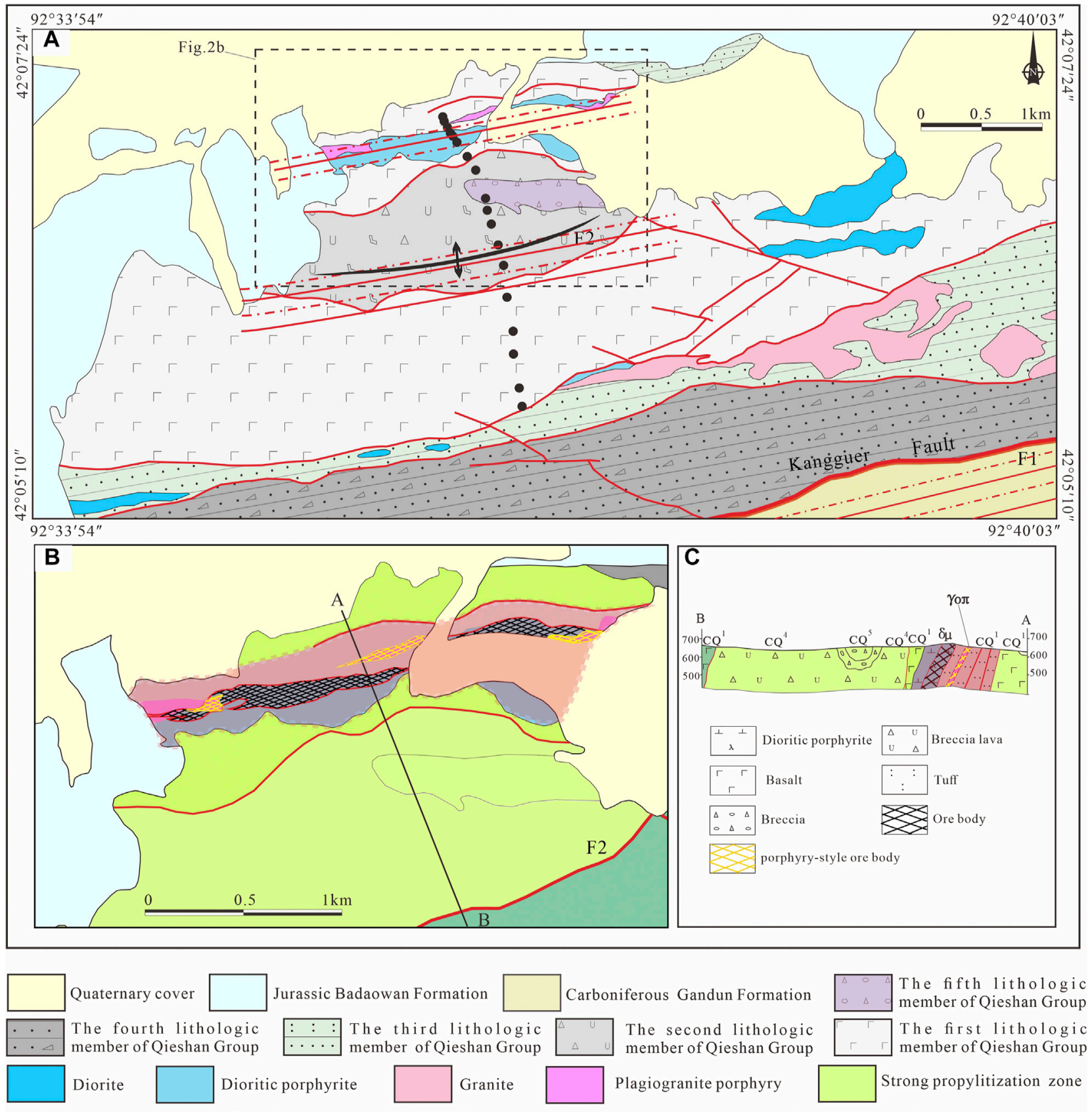

Week propylitization

Quartz-sericite
zone

- Sampling location

FIGURE 2 | (A) Geological map of the porphyry Cu deposit in eastern Tianshan, Xinjiang, NW China (revision according to Ma et al., 2003; Pan et al., 2013). (B) Alteration zonation map of the Tuwu copper ore district. (C) Profile map of the Tuwu copper ore district.

propylitization are chlorite, epidote, quartz, zoisite, calcite, and pyrite, and the rock color is dark green in general (Figures 4A,F; Figure 5A). Chlorite is mainly in the form of interstitial mafic minerals developed between feldspars. The formation of chlorite is related to the late hydrothermal alteration of dark minerals, such as amphibole. Epidote mainly exists discontinuously in the form of veins in faulted structures.
Pyrite grains with particle sizes of $0.01-0.05 \mathrm{~nm}$ exhibit a stellate distribution.

The concentric alteration zones from the interior outward in succession are the potassic zone, the quartz-sericite zone, and the propylitic zone.

The potassic zone is mainly distributed in plagiogranite porphyry and dioritic porphyrite. The alteration mineral 


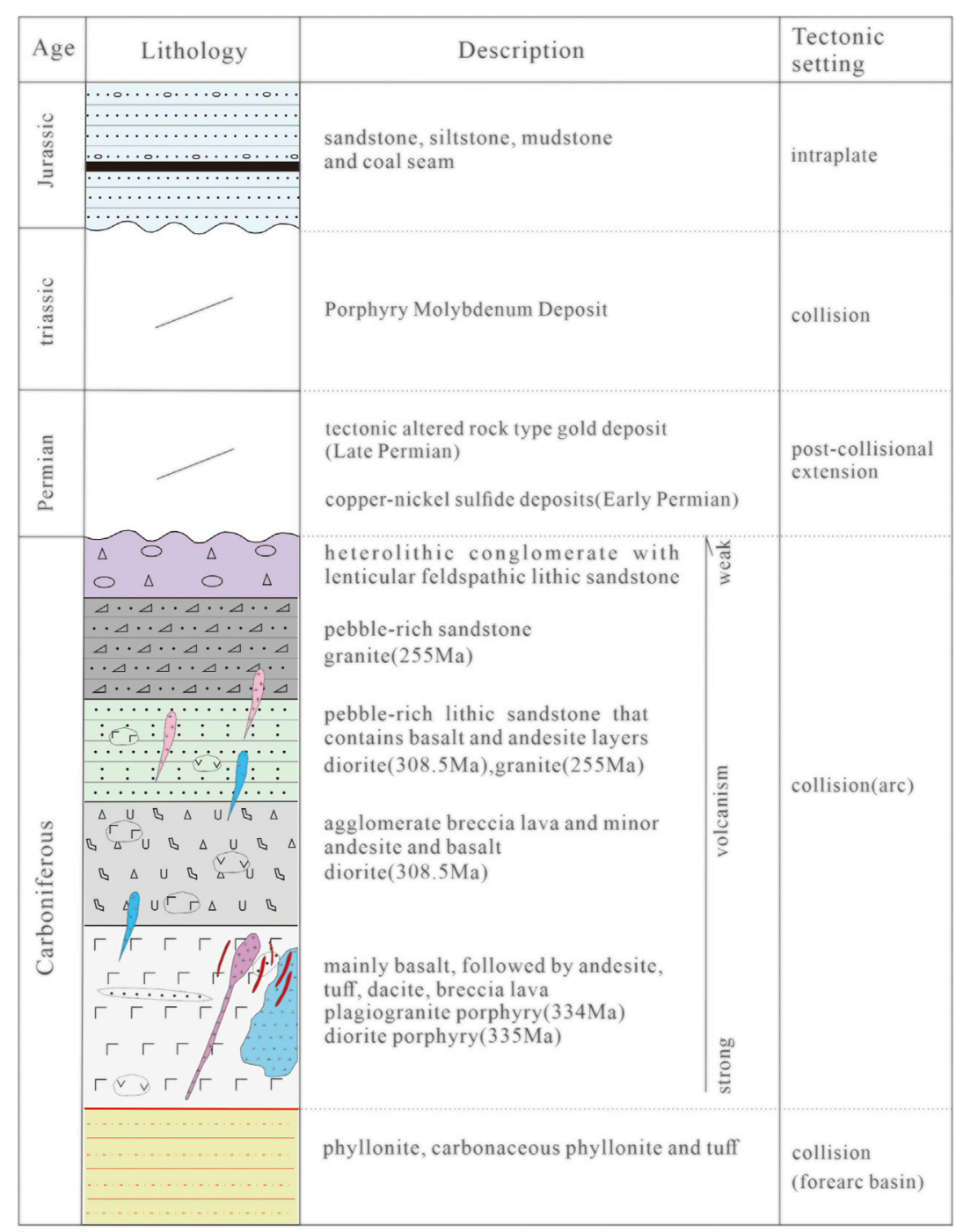

FIGURE 3 | The chronostratigraphic chart of geological events in Tuwu copper ore district.

assemblage of disseminated and vein types includes potassium feldspar, biotite, and magnetite. The fine-grained and scaly biotite originated from biotitization of hornblende and recrystallization of primary biotite. The hydrothermal biotite is poorly preserved and often altered into chlorite, ilmenite, and rutile. Identifying the potassic zone is difficult because it has been altered by later hydrothermal activity. Consequently, the boundary of the potassic zone is not clear. Pyritization, sericitization, and silicification are well developed in plagiogranite porphyry (Figures 4C,H). Plagioclase has been almost completely altered to sericite and fine-grained quartz. Pyrite is also widespread (Figures 5B,C). The alteration assemblage is mainly sericite, pyrite, quartz, chalcopyrite, and calcite with net veins and disseminated forms. The dioritic porphyrite is locally developed and sericitized. Plagioclase has been altered to sericite and minor epidote and albite. The alteration assemblage is sericite, chlorite, pyrite, epidote, and quartz (Figures 4D,I,J; Figures 5D-F).
The strongly propylitized rocks are bright green in color and exhibit strong silicification. The alteration assemblage is mainly epidote, chlorite, quartz, calcite, and pyrite. Quartz, epidote, and chlorite are mainly distributed in dense disseminated and vein forms. The disseminated quartz is mainly associated with propylitic alteration, while the vein quartz that appears to have a hydrothermal filling genesis is an obviously younger overprinting feature. Radial and granular epidote aggregates can be seen in some veins. The basalt near the ore is altered and contains minor pyrite, epidote, chlorite, quartz, calcite, and chalcopyrite veins (Figures 4B,G).

Alterations related to ductile shear deformation are developed in the local area and characterized by intense sericitization and chloritization. The altered minerals show obvious crumpled and directional arrangement. The rocks have augen and cataclastic structures. The plagioclase in the dioritic porphyrite has been altered to scaly sericite and fine-grained quartz. Chlorite is present in the form of blocks and stockwork and well 

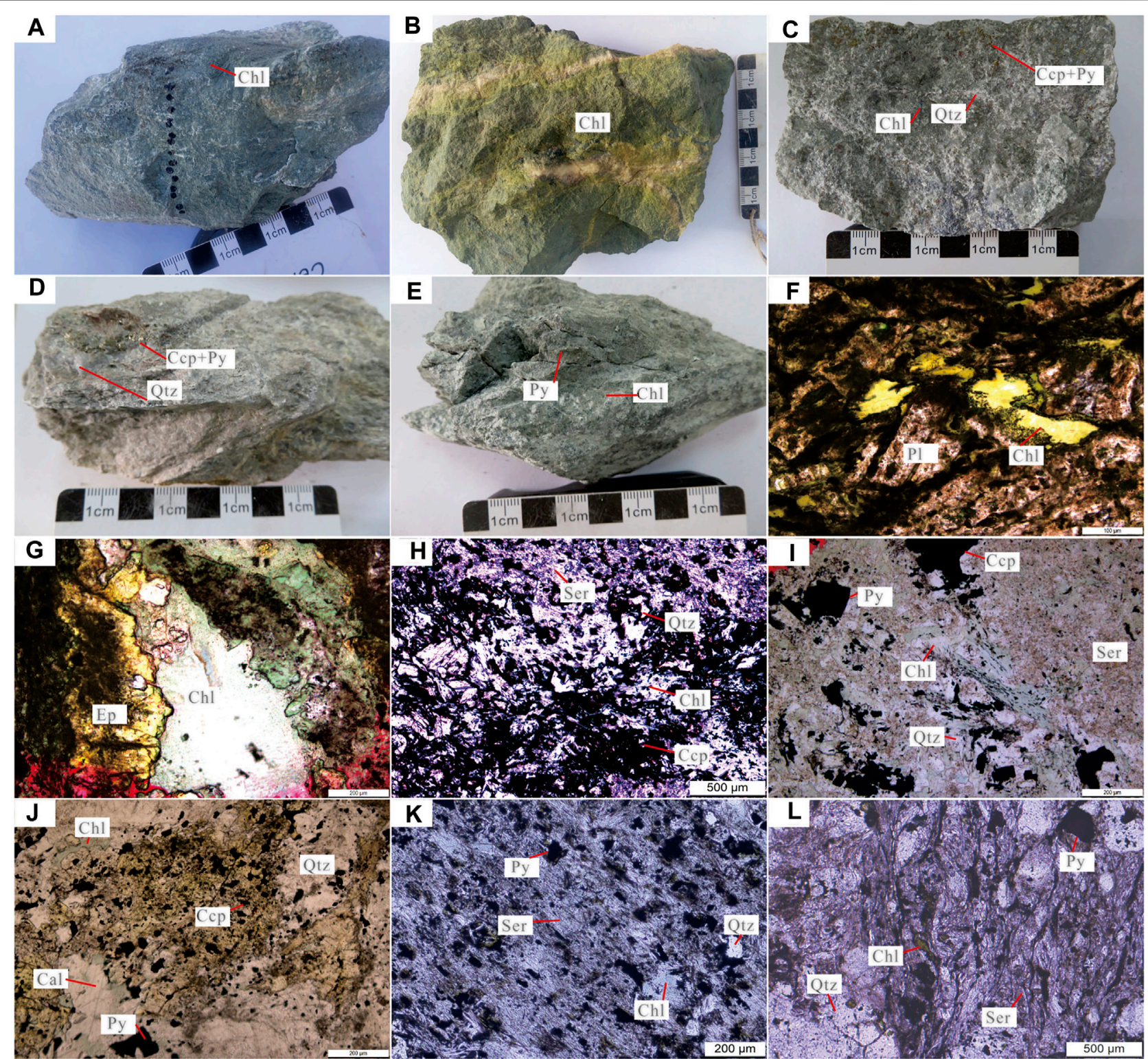

FIGURE 4 | Photomicrographs of the chlorite and specimens of typical ore samples from different mineralization stages of Tuwu porphyry Cu deposit in eastern Tianshan, Xinjiang, NW China. (A) Weakly propylitized basalt; (B) strongly propylitized basalt; (C) pyrite + chalcopyrite + chlorite + sericite in diorite porphyrite; and (D) pyrite + chalcopyrite + silicification development in plagiogranite porphyry; (E) mylonitized diorite porphyrite; (f) weak chlorite development in basalt, Chl-l; (G) strong epidote and chlorite development in basalt, Chl- $\|_{1} ; \mathbf{( H )}$ pyritization, sericitization, silicification, and chloritization of plagiogranite porphyry, Chl- $\mathrm{I}_{2}$; (I) sericite, silicification, and chlorite development in plagiogranite porphyry, Chl- $I_{2} ; \mathbf{( J )}$ chlorite, epidote, pyrite, and calcite development in plagiogranite porphyry, Chl-II $;$ (K) chlorite, chalcopyrite, and sericite in diorite porphyry (chlorite, sericite, and pyrite grains have an oriented arrangement) Chl-III; and (L) chlorite, chalcopyrite, and sericite in diorite porphyry rock with an augen structure, Chl-III. Ccp, chalcopyrite; Py, pyrite; PI, plagioclase; Chl, chlorite; Ep, epidote; Qtz, quartz; Cal, calcite; Ser, sericite.

developed in the local ductile shear zone (Figures 4E,K,L). The altered mineral assemblage is sericite, chlorite, and quartz. The metallic minerals are mainly chalcopyrite and pyrite, which are distributed in disseminated and vein types (Figures 5G,H,I). The alteration zone is related to the rich ore body in this area.

The main supergene alteration mineral assemblage is kaolinite, atacamite, malachite, and hematite.

Previous researchers have conducted a large amount of research on the alteration zonation and metallogenic timing of the Tuwu copper deposit based on the association of alteration minerals, mineral relationships, and crosscutting relationships (Shen et al., 2012; Pan et al., 2013; Wang Y. F. et al., 2016). After extensive research on the petrography of the main rock types exposed in the Tuwu mining area (Figures 2B,C), this study identified four stages of hydrothermal activity, namely, alterations related to volcanic activity (stage I), alterations related to porphyry mineralization (stage II), alterations related to ductile shear deformation (stage III), and alterations related to supergenesis (stage IV) (Figure 6). 

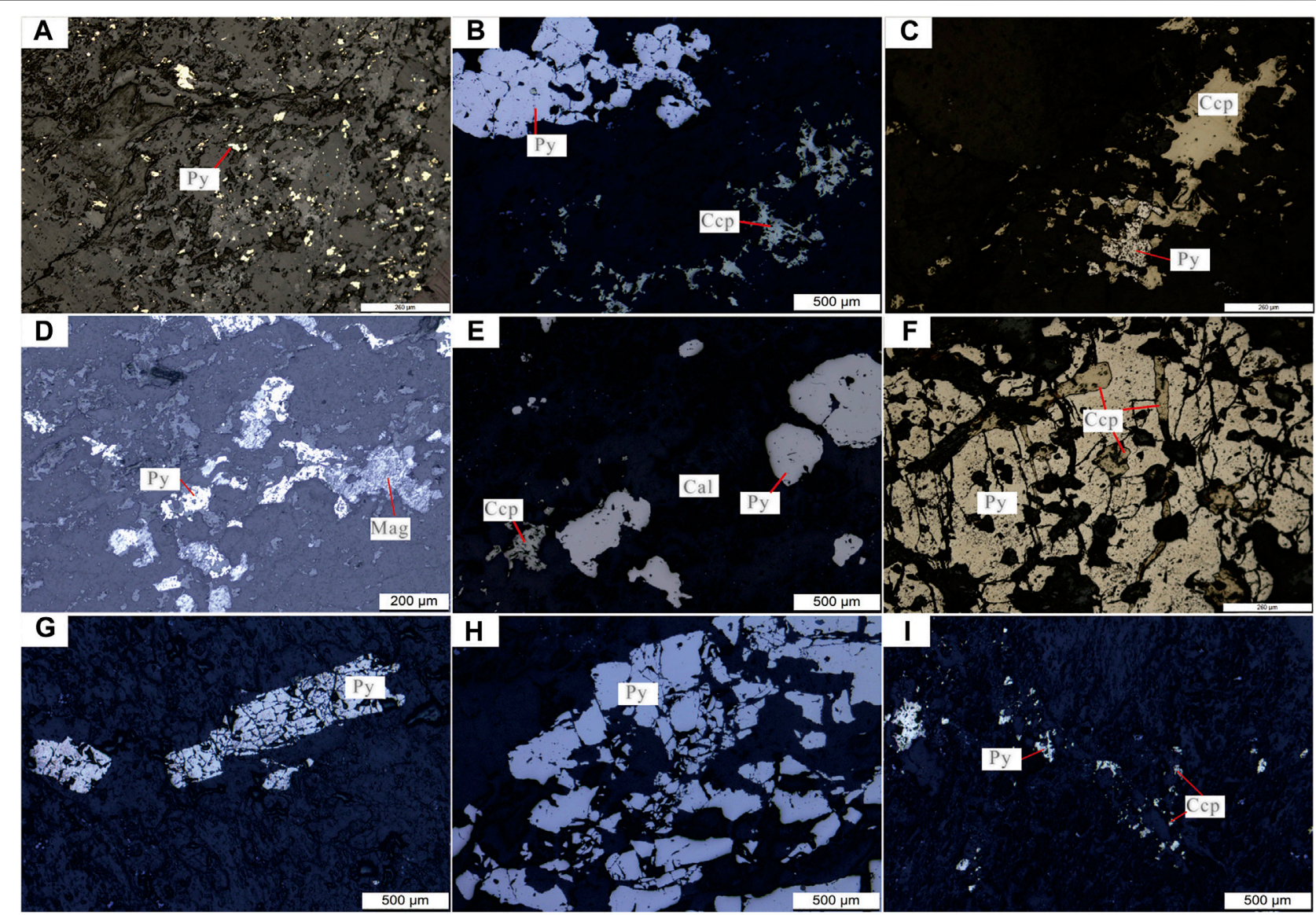

FIGURE 5 | Photomicrographs of pyrite from different mineralization stages in the Tuwu porphyry Cu deposit in eastern Tianshan, Xinjiang, NW China. (A) Disseminated pyrite in basalt with weak propylitization, Py-I; (B) Pyrite associated with chalcopyrite in plagiogranite porphyry, Py- $I_{1}$; (C) pyrite growing along chalcopyrite fissure in plagiogranite porphyry, Py- $\|_{1}$; (D) pyrite associated with magnetite in plagiogranite porphyry, Py- $\|_{2}$; (E) veinlets of pyrite, chalcopyrite, calcite, and silicification, in plagiogranite porphyry, Py- $\|_{2} ; \mathbf{( F )}$ chalcopyrite growing along pyrite fissure in plagiogranite porphyry in diorite porphyry, Py-III; (G) chlorite, sericite, and pyrite in diorite porphyrite, pyrite with crushed structure, Py-III; (H) sericite and pyrite in plagiogranite porphyry, pyrite with crushed structure, Py-III; and (I) chlorite and sericite in diorite porphyrite with pyrite and chalcopyrite vein. Ccp, chalcopyrite; Py, pyrite; Mag, magnetite; Qtz, quartz; Cal, calcite; Ser, sericite.

\section{SAMPLING AND ANALYSIS METHODS}

This study carried out detailed geological observations and profile surveys of outcrops, troughs, open mining pits and drilling samples from the Tuwu mining area. Typical samples of various alteration zones were collected from the area, and thin sections with a thickness of $0.8 \mathrm{~mm}$ were prepared. Two to three typical samples were selected from each alteration zone, and 19 samples were selected for testing and analysis. The mineral types of the tested samples are mainly pyrite Py-I, chlorite Chl- $\mathrm{I}_{1}$ (metasomatic crystallization), and Chl- $\mathrm{I}_{2}$ (precipitation crystallization) related to volcanic activity, pyrite Py-II, and chlorite Chl-II related to porphyry mineralization, pyrite Py-III, and chlorite Chl-III related to ductile shear deformation.

The in situ analysis of chlorite composition was completed in the LA-ICP-MS laboratory of the State Key Laboratory of Ore Deposit Geochemistry, Institute of Geochemistry, Chinese Academy of Sciences. The LA-ICP-MS instrument is composed of an Agilent 7700cs quadrupole ICP-MS instrument connected to a GeoLasPro $193 \mathrm{~nm}$ LA system. The operating conditions of the instrument were as follows: the laser beam width was $44 \mathrm{~nm}$, the frequency of the laser pulse was $6 \mathrm{~Hz}$, and the pulse energy was $0.032-0.105$. The analysis time was $90 \mathrm{~s}$ ( $30 \mathrm{~s}$ for the blank test and $60 \mathrm{~s}$ for the sample analysis). During testing, the LA spot coincided with the electron microprobe analysis spot. The internal standards NIST610 and NIST612 were analyzed once every nine chlorite samples to monitor the instrument deviation. The international standard samples ML3BG and BCR-2G were used as unknown samples to monitor the data quality. Data processing was calibrated by Igor-pro software. The Si content was analyzed by electron microprobe as the internal standard to calibrate the Si content determined by LA-ICP-MS. The analysis results of GOR128-G, BIR-1G, BHVO-2G, ML3B-G, and BCR-2G were in good agreement with their reference values. The analytical error of most elements was less than $10 \%$, although $\mathrm{Cr}, \mathrm{Y}$, and $\mathrm{Zr}$ had errors of $10-15 \%$. 


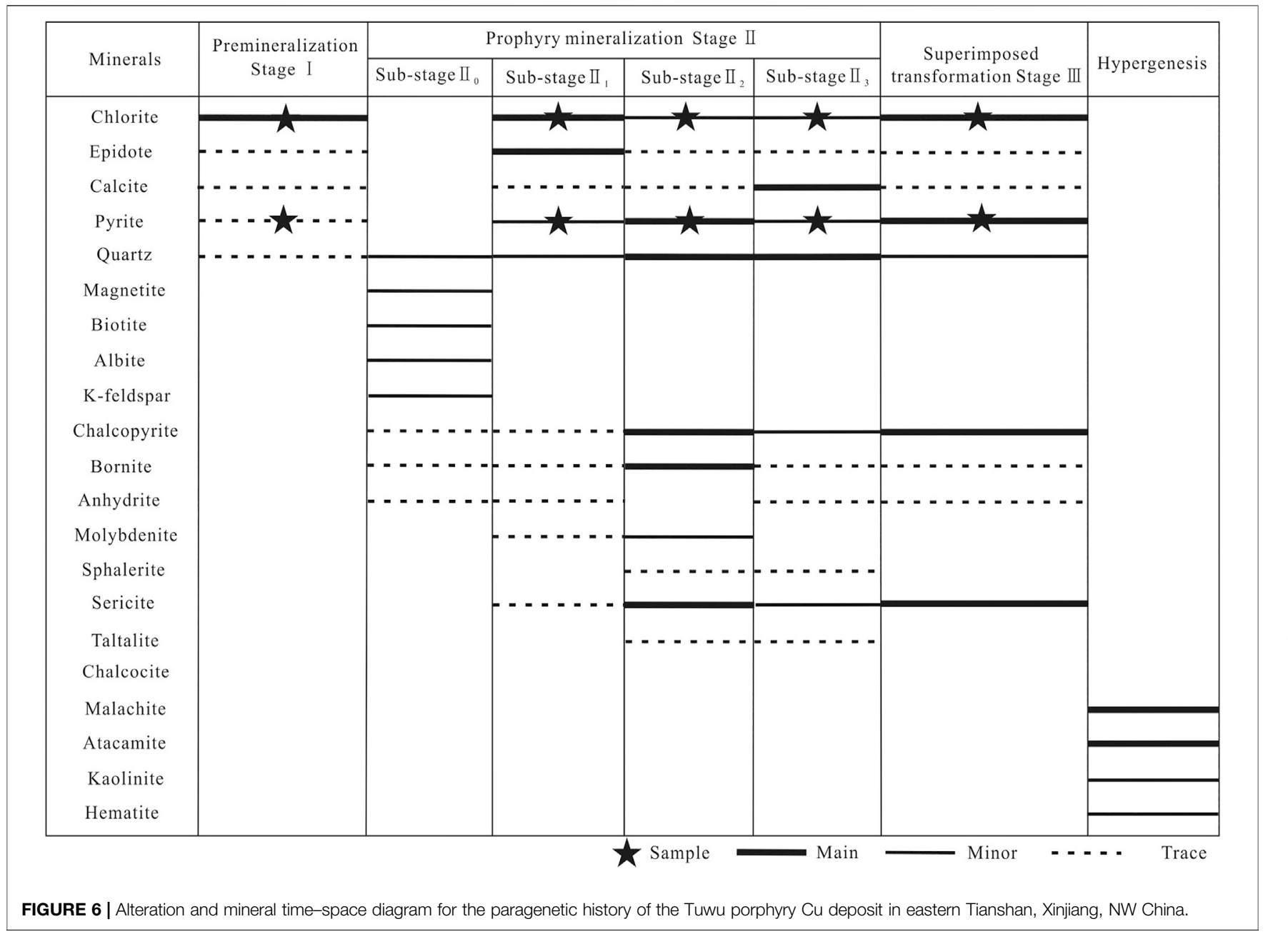

The analysis of pyrite was carried out in the State Key Laboratory of Ore Deposit Geochemistry, Institute of Geochemistry, Chinese Academy of Sciences. The standard sample of the LA system was NWR-213, and the experimental analysis instrument was an Agilent 7700x type quadrupole mass spectrometer. Helium ( $480 \mathrm{ml} / \mathrm{min}$ ) was used as a transport medium for the ablated material, and it was mixed evenly with argon $(900 \mathrm{ml} / \mathrm{min})$ after leaving the ablation compartment. The mixture then entered the ICP-MS instrument, where the element contents were measured. The analysis time was $90 \mathrm{~s}$ (30 s for blank test and $60 \mathrm{~s}$ for sample analysis). The laser beam width was $44 \mathrm{~nm}$, the frequency of the laser pulse was $10 \mathrm{~Hz}$, and the pulse energy was $5 \mathrm{~J} / \mathrm{cm}^{2}$. The analysis method was a multiple external standard-no internal standard method, and the external standards were GSE-1G, MASS, and Py. Data processing was performed with ICPMSDataCal software.

\section{TEST RESULTS}

\section{In Situ Trace Element Composition of Pyrite}

The results of the in situ trace element composition of pyrite in the Tuwu copper mining area are shown in Table 1. Overall, the contents of chalcophile elements $(\mathrm{Cu}, \mathrm{Zn}, \mathrm{Pb}, \mathrm{As}, \mathrm{Se}, \mathrm{Te}, \mathrm{Ge}$, and $\mathrm{Ag}$ ), siderophile elements ( $\mathrm{Cr}, \mathrm{Co}$, and $\mathrm{Ni}$ ), $\mathrm{V}$, and $\mathrm{Ba}$ are all above the limit of detection. The contents of $\mathrm{Sn}, \mathrm{Cd}, \mathrm{Sb}, \mathrm{Au}, \mathrm{Bi}$, and other elements were mostly lower than or near the detection limit (Figures 7, 8).

The results showed that Py-I has the highest total amount of trace elements (average 4,351 ppm) and highest $\mathrm{Co} / \mathrm{Ni}$ ratio (average 33). $\mathrm{Py}-\mathrm{I}$ is rich in $\mathrm{Co}, \mathrm{Ni}$, and $\mathrm{As}$ and poor in $\mathrm{Cu}$ and $\mathrm{Pb}$, and the $\mathrm{Zn}, \mathrm{Au}$, and $\mathrm{Ag}$ contents are below or near the detection limit. The total amount of trace elements of $\mathrm{Py}-\mathrm{II}_{1}$ (average 4,049 ppm) and $\mathrm{Py}-\mathrm{II}_{2}$ (average $544 \mathrm{ppm}$ ) displayed an obvious decrease with the occurrence of porphyry mineralization. Moreover, the $\mathrm{Co} / \mathrm{Ni}$ ratio of $\mathrm{Py}-\mathrm{II}_{1}$ (average 3) and $\mathrm{Py}-\mathrm{II}_{2}$ (average 0.53) also showed a certain decrease. Py$\mathrm{II}_{1}$ and $\mathrm{Py}-\mathrm{II}_{2}$ are rich in $\mathrm{Co}, \mathrm{Ni}$, and $\mathrm{Cu}$, and poor in $\mathrm{As}, \mathrm{Pb}, \mathrm{Zn}$, $\mathrm{Au}$, and Ag. The total amount of trace elements in Py-III ranges from 133 to 6,401 ppm (average $1982 \mathrm{ppm}$ ). Py-III has the lowest $\mathrm{Co} / \mathrm{Ni}$ ratio (average 0.16). This phase features obviously higher mineralization element contents than Py-I, $\mathrm{Py}-\mathrm{II}_{1}$, and $\mathrm{Py}-\mathrm{II}_{2}$, and the contents of elements, such as $\mathrm{Co}, \mathrm{Ni}$, $\mathrm{Cu}, \mathrm{Pb}, \mathrm{As}, \mathrm{Pb}, \mathrm{Zn}, \mathrm{Au}$, and $\mathrm{Ag}$, have extremely uneven distributions (Table 1). 
TABLE 1 | In situ trace element compositions of pyrite for different metallogenic stages of the Tuwu porphyry Cu deposit in eastern Tianshan, Xinjiang, NW China (ug/g).

\begin{tabular}{|c|c|c|c|c|}
\hline \multirow{2}{*}{$\begin{array}{l}\text { Trace } \\
\text { element (ppm) }\end{array}$} & Py-I & Py-II & $\mathrm{Py}-\mathrm{II}_{2}$ & Py-III \\
\hline & $n=3$ (total) & $n=11$ (total) & $n=10$ (total) & $n=6$ (total) \\
\hline V & $0.68-1.11 ; \mathrm{Av}=0.87(n=3)$ & $0.06-32.17 ; A v=7.34(n=11)$ & $0.03-0.29 ; \mathrm{Av}=0.09(n=10)$ & $\begin{array}{c}\text { Mostly bdl; } 0.53-0.53 ; \text { Av } \\
=0.53\end{array}$ \\
\hline $\mathrm{Cr}$ & 3.02-9.07; Av = $5.65(n=3)$ & $1.21-27.41 ; \mathrm{Av}=7.28(n=11)$ & 1.08-18.45; Av = $6.35(n=10)$ & 2.25-20.96; Av = 7.56 \\
\hline Co & $\begin{array}{c}288.5-6,417.17 ; A v=3,027.09 \\
(n=3)\end{array}$ & $\begin{array}{c}115.7-6,339.2 ; A v=2,260.87 \\
(n=11)\end{array}$ & 5.73-318.06; Av = $91.40(n=10)$ & $0.65-415.9 ; A v=75.21$ \\
\hline $\mathrm{Ni}$ & 25.38-105.89; $A v=71.9(n=3)$ & $\begin{array}{c}27.58-3,396.17 ; A v=1,635.83 \\
(n=11)\end{array}$ & $\begin{array}{c}11.19-1,623.02 ; A v=325.23 \\
(n=10)\end{array}$ & 47.38-1,298.36; Av = 509.45 \\
\hline $\mathrm{Cu}$ & 8.95-50.68; Av = $23.16(n=3)$ & $0.66-336.25 ; \mathrm{Av}=86.28(n=10)$ & 2.96-43.6; Av = $16.38(n=10)$ & $5.94-4,133.8 ; A v=950.33$ \\
\hline $\mathrm{Zn}$ & $0.1-0.7 ; \mathrm{Av}=0.4(n=2)$ & $0.13-10.42 ; A v=3.13(n=6)$ & $0.29-2.61 ; A v=1.17(n=8)$ & $\begin{array}{c}\text { Mostly bdl; } 8.84-8.84 ; \text { Av } \\
=8.84\end{array}$ \\
\hline $\mathrm{Ge}$ & $7.87-11.69 ; A v=9.15(n=3)$ & $6.75-12.88 ; A v=9.28(n=11)$ & 7.23-13.85; Av = $9.24(n=10)$ & $6.17-10.85 ; A v=8.38$ \\
\hline As & $\begin{array}{c}50.73-2,933.98 ; A v=1,072.03 \\
(n=3)\end{array}$ & $0.27-21.14 ; \mathrm{Av}=5.03(n=10)$ & $0.01-58.85 ; \mathrm{Av}=16.95(n=8)$ & $10.1-1817.01 ; A v=913.56$ \\
\hline $\mathrm{Se}$ & 87.3-167.07; Av = $133.16(n=3)$ & $21.15-82.59 ; A v=39.62(n=11)$ & 41.95-174.06; Av = $71.49(n=10)$ & 19.65-137.42; Av = 68.10 \\
\hline $\mathrm{Ag}$ & $0.02-0.16 ; \mathrm{Av}=0.11(n=3)$ & $0.02-0.57 ; \mathrm{Av}=0.13(n=9)$ & $0.02-1.4 ; A v=0.37(n=9)$ & $0.02-20.22 ; A v=5.21$ \\
\hline $\mathrm{Cd}$ & $0.22-0.33 ; A v=0.27(n=3)$ & $0.1-0.44 ; \mathrm{Av}=0.24(n=6)$ & $0.01-0.49 ; \mathrm{Av}=0.17(n=7)$ & $0.39-1.41 ; A v=0.69$ \\
\hline Sn & bdl & $0.01-3.63 ; A v=0.75(n=8)$ & $0.01-0.49 ; \mathrm{Av}=0.26(n=6)$ & $0.12-1.07 ; A v=0.46$ \\
\hline $\mathrm{Sb}$ & $0.22-0.22 ; A v=0.22(n=2)$ & $0.01-0.37 ; A v=0.09(n=6)$ & $0.06-4.8 ; A v=0.73(n=8)$ & $0.08-14.34 ; A v=3.66$ \\
\hline $\mathrm{Te}$ & $0.43-8.24 ; A v=3.42(n=3)$ & $0.02-0.74 ;$ Av $=0.42(n=8)$ & $0.01-3.05 ; \mathrm{Av}=0.68(n=8)$ & $0.61-12.15 ; A v=5.57$ \\
\hline $\mathrm{Ba}$ & $0.05-0.42 ; A v=0.19(n=3)$ & $0.03-8.12 ; \mathrm{Av}=2.18(n=8)$ & $0.06-0.91 ; \mathrm{Av}=0.25(n=9)$ & $0.15-0.3 ; A v=0.21$ \\
\hline $\mathrm{Au}$ & $0.03-0.03 ; A v=0.03(n=2)$ & $0.01-0.04 ; \mathrm{Av}=0.02(n=7)$ & $0.01-0.1 ; \mathrm{A} v=0.03(n=7)$ & $0.01-4.91 ; A v=1.24$ \\
\hline $\mathrm{Pb}$ & 1.93-6.86; Av = $3.93(n=3)$ & $0.02-3.41 ; A v=1.16(n=11)$ & $0.04-27.01 ; A v=5.99(n=10)$ & $0.66-164.32 ; A v=48.61$ \\
\hline $\mathrm{Bi}$ & 0.09-0.19; Av = $0.12(n=3)$ & $0.02-0.79 ; \mathrm{Av}=0.34(n=8)$ & $0.01-6.2 ; \mathrm{Av}=1.28(n=8)$ & $0.01-13.82 ; A v=3.39$ \\
\hline $\mathrm{Co} / \mathrm{Ni}$ & 11.37-60.60; Av = $33.37(n=3)$ & $0.09-9.94 ; \mathrm{Av}=2.71(n=11)$ & $0.12-2.02 ; A v=0.53(n=10)$ & $0.003-0.57 ; A v=0.16$ \\
\hline Total content & $\begin{array}{c}\text { 478.09-9,692.23; Av = 4,351.34 } \\
(n=3)\end{array}$ & $\begin{array}{c}\text { 594-7,839.08; Av }=4,049.19 \\
(n=11)\end{array}$ & $\begin{array}{c}125.64-2028.18 ; A v=543.68 \\
(n=10)\end{array}$ & $\begin{array}{l}\text { 132.52-6,401.02; Av }= \\
1982.41\end{array}$ \\
\hline
\end{tabular}

Note: Av stands for average and bdl stands for below the detection limit.

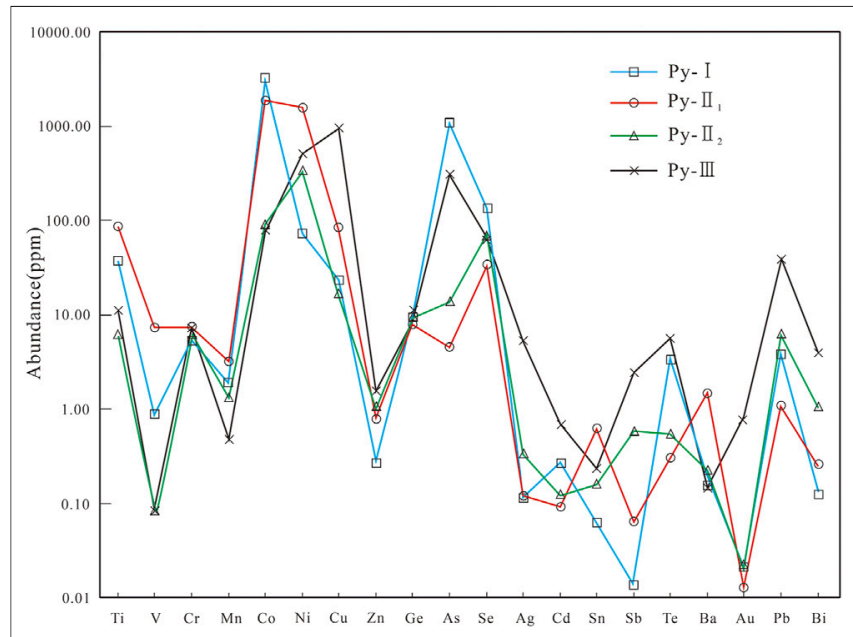

FIGURE 7 | The average trace element composition of pyrite in different mineralization stages in the Tuwu porphyry $\mathrm{Cu}$ deposit in eastern Tianshan, Xinjiang, NW China.

\section{In Situ Trace Element Composition of Chlorite}

The results of the in situ trace element composition of chlorite in the Tuwu copper mining area are shown in Table 2. To ensure that the mineral in the experiment is chlorite, the chlorite are filtered based on the standard of $\omega\left(\mathrm{Na}_{2} \mathrm{O}+\mathrm{K}_{2} \mathrm{O}+\mathrm{CaO}\right)<0.5 \%$
(Foster, 1962; Zang and Fyfe, 1995). According to the Fe-Si classification of chlorite, the chlorite samples are mainly clinochlore and pyrochlore (unpublished data).

In this study, the data show that the total rare earth element (REE) content of chlorite from different stages is above the detection limit, thus, REEs are selected for research. The content of REEs in Chl- $\mathrm{I}_{1}$ ranges from 0.03 to $17.62 \mathrm{ppm}$, and the REE patterns are characterized by obvious enrichment of light REEs over heavy REEs (LREE/HREE = $0.12-5.95)$, negative $\mathrm{Eu}$ anomalies $(\delta \mathrm{Eu}=0.223-0.866)$, and no Ce anomalies $(\delta \mathrm{Ce}=0.53-2.11)$. The results of $(\mathrm{La} / \mathrm{Sm}) \mathrm{n}$ $(0.14-1.33)$ and $(\mathrm{Gd} / \mathrm{Yb}) \mathrm{n}(0.95-4.70)$ indicate that the internal fractionation of LREEs and HREEs is weak (Figure 9A).

The content of REEs in Chl- $\mathrm{I}_{2}$ ranges from 0.02 to $0.71 \mathrm{ppm}$, and the REE patterns are mainly characterized by the enrichment of LREEs (LREE/HREE $=0.18-3.91)$, positive Eu anomalies ( $\delta \mathrm{Eu}$ $=1.0-3.78)$, and weak $\mathrm{Ce}$ anomalies $(\delta \mathrm{Ce}=0.54-2.01)$. The results of $(\mathrm{La} / \mathrm{Sm}) \mathrm{n}(0.07-1.81)$ and $(\mathrm{Gd} / \mathrm{Yb}) \mathrm{n}(0.22-5.61)$ indicate that the internal fractionation of LREEs and HREEs is relatively weak (Figure 9B).

The content of REEs in Chl- $\mathrm{II}_{1}$ ranges from 0.056 to $0.77 \mathrm{ppm}$, and the REE patterns are characterized by the relative enrichment of HREEs (LREE/HREE $=0.07-0.59)$, positive Eu anomalies $(\delta \mathrm{Eu}$ $=1.08-1.62)$, and weakly negative $\mathrm{Ce}$ anomalies $(\delta \mathrm{Ce}=$ $0.43-1.52)$. The results of $(\mathrm{La} / \mathrm{Sm}) \mathrm{n}(0.11-0.497)$ and $(\mathrm{Gd} / \mathrm{Yb})$ $\mathrm{n}(1.1-12.02)$ indicate that the internal fractionation of LREEs and HREEs is stronger (Figure 9C). 

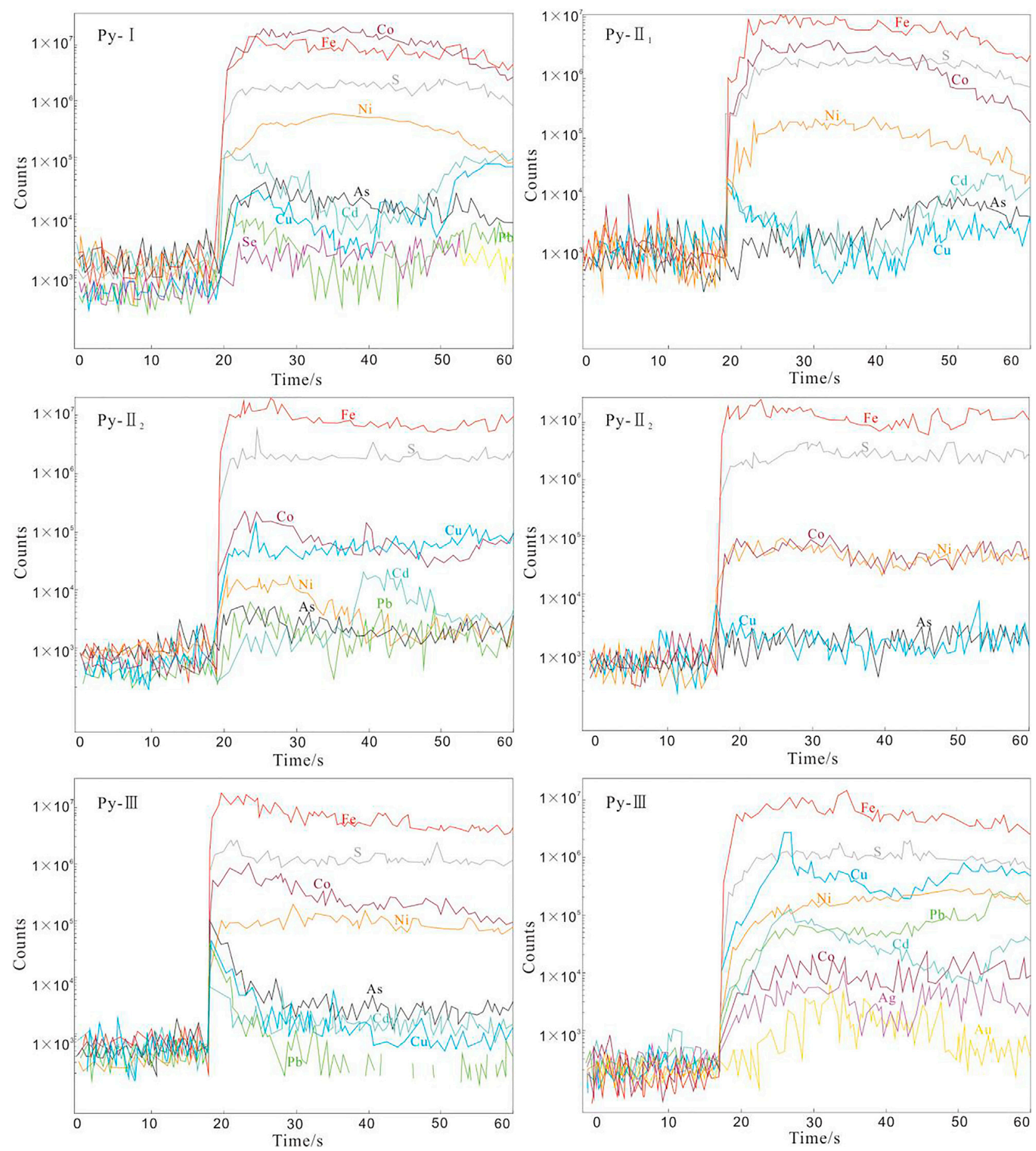

FIGURE 8 | LA-ICP-MS ablation curves of pyrite in different mineralization stages of the Tuwu porphyry Cu deposit in eastern Tianshan, Xinjiang, NW China.

The content of REEs in Chl- $\mathrm{II}_{2}$ ranges from 0.02 to $3.42 \mathrm{ppm}$, and the REE patterns are characterized by the relative enrichment of HREEs (LREE/HREE $=0.11-1.19$ ), weak positive Eu anomalies $(\delta \mathrm{Eu}=1.02-2.52)$, and positive Ce anomalies $(\delta \mathrm{Ce}=0.52-1.95)$. The results of $(\mathrm{La} / \mathrm{Sm}) \mathrm{n}(0.07-0.71)$ and $(\mathrm{Gd} / \mathrm{Yb}) \mathrm{n}(0.502-11.60)$ indicate that the internal fractionation of LREEs and HREEs is strong (Figure 9D).

The content of REEs in Chl- $\mathrm{II}_{3}$ ranges from 0.05 to $3.34 \mathrm{ppm}$, and the REE patterns are characterized by the relative enrichment of HREEs (LREE/HREE $=0.08-0.85$ ), negative Eu to weakly 
TABLE 2 | In situ trace element composition and characteristic REE values of chlorite for different metallogenic stages of the Tuwu porphyry Cu deposit in eastern Tianshan, Xinjiang, NW China ( $\mu g / g)$.

Trace

element

$n=17$
(tota)

$$
\begin{aligned}
& n=17 \\
& \text { (total) }
\end{aligned}
$$

76.46-1,050.02; $\mathrm{Av}=498.1$ $(n=17)$

66.64-660.13; $\mathrm{Av}=252.8$ $(n=17)$

$\mathrm{Ca}$

101.30-2,525.45; Av = 828.3 $(n=17)$

12.22-159.33; $\mathrm{Av}=29.64$

$$
(n=17)
$$

44.63-210.72; Av = 115.75 $(n=17)$

1946.41-3,466.17; Av =

2,275.41 $(n=17)$

153.95-387.96; Av = 309.24 $(n=17)$

1.81-345.91; $\mathrm{Av}=98.07$ $(n=17)$

190.46-411.24; $\mathrm{Av}=257.89$ $(n=17)$

$0.18-0.76 ; \mathrm{Av}=0.413(n=6)$ 0.002-0.025; $\mathrm{Av}=0.012(n=6)$

$0.002-0.456 ; \mathrm{Av}=0.085$

$$
(n=14)
$$

$0.001-2.132 ; \mathrm{Av}=0.208$

$$
(n=16)
$$

$0.003-6.37 ; \mathrm{Av}=0.566(n=17)$

$0.002-0.085 ; \mathrm{Av}=0.017$ $(n=13)$

$0.007-0.847 ; \mathrm{Av}=0.153$ $(n=12)$

$0.0009-0.12 ; \mathrm{Av}=0.017$

${\text { Chl- } \mathbf{2}_{2}}^{n=15}$
(total)

76.75-1,413.61; Av = 562.06 $(n=15)$

79.72-596.30; $\mathrm{Av}=274.13$ $(n=15)$ 1.79-29.16; $\mathrm{Av}=10.94(n=17)$

$0.01-0.159 ; \mathrm{Av}=0.088(n=16)$ $0.053-0.107 ; \mathrm{Av}=0.062(n=6)$

$0.001-0.93 ; \mathrm{Av}=0.101(n=14)$

$0.008-4.56 ; \mathrm{Av}=0.506(n=14)$

$0.007-1.06 ; \mathrm{Av}=0.155(n=12)$ $(n=16)$ $(n=15)$

10.96-573.20; $\mathrm{Av}=71.45$

$$
(n=15)
$$

35.38-188.80; Av = 99.53 $(n=15)$

1,376.12-2,973.11; Av = $2099.84(n=15)$

147.21-397.03; Av = 301.59 $(n=15)$

3.11-215.25; $\mathrm{Av}=106.21$ $(n=15)$

185.61-293.06; $\mathrm{Av}=256.57$ $(n=15)$

$0.02-1.11 ; \mathrm{Av}=0.506(n=7)$

$0.86-7.49 ; \mathrm{Av}=4.79(n=15)$

0.13-0.023; Av = $0.071(n=15)$

$0.02-0.09 ; \mathrm{Av}=0.052(n=5)$

$0.005-0.02 ; \mathrm{Av}=0.011(n=6)$

0.0004-0.62; Av $=0.193$

$$
(n=12)
$$

0.0009-0.043; $\mathrm{Av}=0.012$ $(n=15)$

0.0008-0.18; Av $=0.037$ $(n=12)$

$0.0007-0.02 ; \mathrm{Av}=0.059$ $(n=15)$

0.0056-0.048; $\mathrm{Av}=0.026$ $(n=12)$

0.0005-0.026; $\mathrm{Av}=0.013$ $(n=11)$

0.0022-0.064; Av $=0.026$ $(n=15)$

0.0009-0.014; $\mathrm{Av}=0.006$ $(n=11)$
269.87-1,274.41; Av = 637.36

86.82-2,104.71; $\mathrm{Av}=581.74 \quad 219.25-1,550.54 ; \mathrm{Av}=455.25$

$0.002-0.021 ; \mathrm{Av}=0.01(n=12) \quad 0.0033-0.02 ; \mathrm{Av}=0.011(n=17)$

11.07-176.09; $\mathrm{Av}=37.54$

\begin{tabular}{c} 
Chl-II \\
\hline$n=18$ \\
(total)
\end{tabular}
$(n=18)$

27.8-398.35; $A v=180.18$ $(n=18)$

(2)

ChI-II

(total)

$24.09-1,346.2 ; A v=512.28$ $(n=10)$

12.58-804.58; Av = 198.13 $(n=10)$

68.92-569.66; $\mathrm{Av}=246.97$ $(n=8)$

$$
(n=18)
$$

38.78-181.6: Av $=103.62$ $(n=18)$

2,368.23-3,662.86; Av

$2,711.42(n=18)$

251.76-371.36; Av = 329.01 $(n=18)$

0.02-388.38; Av = $68.54(n=18)$

157.33-597.87; Av $=251.98$ $(n=18)$

$0.02-1.05 ; A v=0.576(n=15)$

$1.83-7.1 ; A v=4.45(n=18)$

19-486.8; Av = 165.92

$$
(n=10)
$$

28.67-933.29; $\mathrm{Av}=229.91$ $(n=10)$

380.31-2048.76; $\mathrm{Av}=$

$1,505.32(n=10)$

80.73-477.43; Av = 325.56 $(n=10)$

0.05-102.99; $\mathrm{Av}=37.96$ $(n=7)$

40.31-395.41; $A v=192.57$ $(n=10)$

2.98-3.66; $\mathrm{Av}=3.33(n=3)$

0.51-58.69; $\mathrm{Av}=12.79$ $(n=10)$

0.01-0.09; $A v=0.056(n=9)$

$0.0064-0.065 ; \mathrm{Av}=0.037$ $(n=14)$

0.0012-0.056; $A v=0.022$ $(n=15)$

0.001-0.0186; $A v=0.067$ $(n=14)$

$0.0018-0.014 ; A v=0.008$ $(n=16)$

0.0038-0.067; $\mathrm{Av}=0.027$ $(n=18)$

0.0008-0.016; $A v=0.007$ $(n=15)$

$0.0086-0.11 ; \mathrm{Av}=0.038(n=16)$

0.0039-0.04; $\mathrm{Av}=0.024(n=16)$

0.0093-0.084; Av = 0.048 $(n=18)$

$0.0028-0.024 ; \mathrm{Av}=0.01(n=16)$
$0.007-0.013 ; \mathrm{Av}=0.01(n=3)$

$0.001-0.20 ; A v=0.068$

$$
(n=10)
$$

$0.001-0.01: A v=0.008$ $(n=10)$

$0.002-0.1 ; \mathrm{Av}=0.03(n=10)$

$0.001-0.01 ; A v=0.005$ $(n=10)$

$0.008-0.12 ; A v=0.047(n=8)$ $0.006-0.05 ; \mathrm{Av}=0.02(n=8)$

$0.004-0.042 ; \mathrm{Av}=0.017$ $(n=8)$

$0.0001-0.125 ; \mathrm{Av}=0.045$ $(n=7)$

$0.0008-0.045 ; \mathrm{Av}=0.016$

$0.07-0.11 ; \mathrm{Av}=0.09(n=2) \quad 0.022-0.15 ; \mathrm{Av}=0.075(n=5)$

\begin{tabular}{c} 
Chl-II \\
\hline$n=13$ \\
(total)
\end{tabular}

20.07-864.1; Av = 202.91 $(n=13)$

$11.51-2,164.91 ; \mathrm{Av}=337.54$ $(n=13)$

10.25-849.34; Av = 203.19 $(n=13)$

21.64-1,055.86; Av = 334.43 $(n=13)$

127.41-780.29; $\mathrm{Av}=363.64$ $(n=13)$

348.55-2,715.22; Av =

$1,139.89(n=13)$ $(n=13)$

$0.01-1.03 ; \mathrm{Av}=0.288(n=10)$

57.63-154.24; $\mathrm{Av}=106.17$ $(n=13)$

2.02-4.15; $\mathrm{Av}=3(n=5)$

$0.37-121.19 ; \mathrm{Av}=17.18$ $(n=13)$

$0.012-0.099 ; \mathrm{Av}=0.045$ $(n=12)$

$0.004-0.027 ; \mathrm{Av}=0.013$ $(n=6)$

0.003-0.0302; $\mathrm{Av}=0.072$ $(n=10)$

$0.002-0.045 ; A v=0.019$ $(n=11)$

$0.006-0.22 ; \mathrm{Av}=0.056(\mathrm{n}$ $=12$ )

$0.005-0.03 ; \mathrm{Av}=0.011$

$(n=10)$
$0.008-0.14 ; \mathrm{Av}=0.07(n=10)$

$0.006-0.1 ; A v=0.034(n=11)$

$0.002-0.04 ; \mathrm{Av}=0.015$ $(n=11)$

$0.005-0.18 ; \mathrm{Av}=0.065$ $(n=11)$

$0.0003-0.04 ; \mathrm{Av}=0.015$ $(n=11)$ (total)

42-96.48; Av = $66.36(n=6)$

63.82-483.25; $A v=144.47$ $(n=6)$

140-553.39; Av = 310.82 ( $=6$ )

177.9-572.4; Av = $299.68(\mathrm{n}$

53.74-254.63:Av $=214.70(n$ $=6$ )

951.67-2,853.68; Av

$2.68-313.44 ; A v=284.64$ $(n=6)$

$0.04-1.2 ; \mathrm{Av}=0.64(n=6)$

154.04-188.16; Av = 168.21 $(n=6)$

$$
\begin{gathered}
n=6) \\
\text { bdl }
\end{gathered}
$$

0.71-2.02; $\mathrm{Av}=1.42(n=6)$

$0.04-0.34 ; \mathrm{Av}=0.12(n=6)$

bdl

bdl

$0.11-0.25 ; \mathrm{Av}=0.2(n=3)$

$0.0004-0.0758 ; \mathrm{Av}=0.03$ $(n=6)$

$0.011-0.244 ; \mathrm{Av}=0.099(n=6)$

$0.001-0.052 ; \mathrm{Av}=0.022(n=5)$

$0.029-0.351 ; \mathrm{Av}=0.144(n=5)$

$0.007-0.106 ; A v=0.053(n=5)$

$0.011-0.047 ; \mathrm{Av}=0.019(n=5)$

$0.008-0.1071 ; \mathrm{Av}=0.048$ $(n=5)$

$0.0001-0.025 ; \mathrm{Av}=0.0068$

$$
(n=6)
$$

(Continued on following page) 
TABLE 2 | (Continued) In situ trace element composition and characteristic REE values of chlorite for different metallogenic stages of the Tuwu porphyry Cu deposit in eastern Tianshan, Xinjiang, NW China ( $\mu$ g/g).

\begin{tabular}{|c|c|c|c|c|c|c|}
\hline \multirow{2}{*}{$\begin{array}{l}\text { Trace } \\
\text { element }\end{array}$} & \multicolumn{2}{|r|}{ Chl-I 2} & Chl-II & ChI-II 2 & Chl-II 3 & Chl-III \\
\hline & $\begin{array}{l}n=17 \\
\text { (total) }\end{array}$ & $\begin{array}{l}n=15 \\
\text { (total) }\end{array}$ & $\begin{array}{l}n=18 \\
\text { (total) }\end{array}$ & $\begin{array}{l}n=10 \\
\text { (total) }\end{array}$ & $\begin{array}{l}n=13 \\
\text { (total) }\end{array}$ & $\begin{array}{l}n=6 \\
\text { (total) }\end{array}$ \\
\hline Dy & $\begin{array}{c}0.0007-0.672 ; A v=0.115 \\
(n=14)\end{array}$ & $\begin{array}{c}0.0008-0.088 ; A v=0.044 \\
(n=12)\end{array}$ & $0.039-0.11 ; \mathrm{Av}=0.078(n=17)$ & $0.003-0.454 ; \mathrm{Av}=0.11(n=7)$ & $\begin{array}{c}0.001-0.43 ; A v=0.116 \\
(n=13)\end{array}$ & $\begin{array}{c}0.016-0.109 ; A v=0.054 \\
(n=50\end{array}$ \\
\hline Ho & $\begin{array}{c}0.002-0.139 ; A v=0.028 \\
(n=15)\end{array}$ & $\begin{array}{c}0.0001-0.035 ; A v=0.01 \\
(n=14)\end{array}$ & $\begin{array}{c}0.0085-0.042 ; A v=0.021 \\
(n=16)\end{array}$ & $\begin{array}{c}0.001-0.134 ; A v=0.027 \\
(n=8)\end{array}$ & $\begin{array}{c}0.009-0.12 ; A v=0.031 \\
(n=13)\end{array}$ & $\begin{array}{c}0.0076-0.033 ; \mathrm{Av}=0.013 \\
(n=5)\end{array}$ \\
\hline Er & $0.002-0.43 ; \mathrm{Av}=0.085(n=16)$ & $\begin{array}{c}0.0028-0.10 ; A v=0.037 \\
(n=13)\end{array}$ & $\begin{array}{c}0.0058-0.145 ; A v=0.071 \\
(n=18)\end{array}$ & $\begin{array}{c}0.005-0.796 ; A v=0.191 \\
(n=10)\end{array}$ & $0.005-0.69 ; A v=0.17(n=11)$ & $0.006-0.069 ; \mathrm{Av}=0.03(n=6)$ \\
\hline $\mathrm{Tm}$ & $\begin{array}{c}0.0003-0.045 ; A v=0.012 \\
(n=16)\end{array}$ & $\begin{array}{c}0.0008-0.017 ; A v=0.007 \\
(n=9)\end{array}$ & $0.0018-0.015 ; A v=0.01(n=18)$ & $\begin{array}{c}0.0008-0.13 ; A v=0.027 \\
(n=7)\end{array}$ & $\begin{array}{c}0.0003-0.124 ; A v=0.029 \\
(n=11)\end{array}$ & $0.001-0.009 ; A v=0.004(n=6)$ \\
\hline $\mathrm{Yb}$ & $0.005-0.25 ; \mathrm{Av}=0.073(n=17)$ & $\begin{array}{c}0.0059-0.21 ; A v=0.054 \\
(n=12)\end{array}$ & $\begin{array}{c}0.0236-0.269 ; A v=0.087 \\
(n=18)\end{array}$ & $\begin{array}{c}0.0001-1.175 ; A v=0.206 \\
(n=9)\end{array}$ & $0.009-1.094 ; A v=0.217(=13)$ & $0.006-0.027 ; A v=0.018(n=5)$ \\
\hline Lu & $\begin{array}{c}0.001-0.034 ; A v=0.011 \\
(n=14)\end{array}$ & $\begin{array}{c}0.0008-0.029 ; A v=0.01 \\
(n=10)\end{array}$ & $\begin{array}{c}0.0007-0.037 ; A v=0.013 \\
(n=18)\end{array}$ & $0.002-0.23 ; A v=0.048(n=7)$ & $\begin{array}{c}0.0009-0.198 ; A v=0.042 \\
(n=12)\end{array}$ & $\begin{array}{c}0.0003-0.01 ; A v=0.0004 \\
(n=6)\end{array}$ \\
\hline $\mathrm{TI}$ & $\begin{array}{c}0.0008-0.011 ; A v=0.006 \\
(n=5)\end{array}$ & $0.0009-0.01 ; \mathrm{Av}=0.006(n=6)$ & $\begin{array}{c}0.0003-0.0089 ; A v=0.0045 \\
(n=10)\end{array}$ & $0.005-0.05 ; \mathrm{Av}=0.02(n=3)$ & $\begin{array}{c}0.004-0.125 ; A v=0.029 \\
(n=6)\end{array}$ & bdl \\
\hline$\Sigma$ REE & $0.03-17.62 ; A v=1.8$ & $0.02-0.71 ; A v=0.248$ & $0.056-0.77 ; \mathrm{Av}=0.425$ & $0.02-3.42 ; A v=0.66$ & $0.05-3.34 ; \mathrm{Av}=0.808$ & $0.04-1.27 ; \mathrm{Av}=0.49$ \\
\hline LREE/HREE & $0.12-5.95 ; A v=1.32$ & $0.18-3.91 ; A v=0.96$ & $0.07-0.59 ; A v=0.296$ & $0.11-1.19 ; \mathrm{Av}=0.64$ & $0.08-0.85 ; A v=0.55$ & $1.26-3.17 ; \mathrm{Av}=1.75$ \\
\hline (La/Sm) n & $0.14-1.33 ; A v=0.70$ & $0.07-1.81 ; A v=0.64$ & $0.11-0.497 ; A v=0.268$ & $0.07-0.71 ; A v=0.298$ & $0.124-0.78 ; A v=0.37$ & $0.2-0.88 ; A v=0.52$ \\
\hline$(G d / Y b) n$ & $0.95-4.70 ; \mathrm{Av}=1.90$ & $0.22-5.61 ; A v=2.28$ & $1.1-12.02 ; \mathrm{Av}=3.33$ & $0.502-11.60 ; A v=3.28$ & $0.51-7.38 ; A v=2.99$ & $1.8-8.08 ; \mathrm{Av}=4.84$ \\
\hline$(\mathrm{La} / \mathrm{Yb}) \mathrm{n}$ & $0.066-6.16 ; A v=1.09$ & $0.09-1.09 ; \mathrm{Av}=0.31$ & $0.01-0.09 ; A v=0.06$ & $0.01-8.11 ; \mathrm{Av}=1.05$ & $0.02-1.93 ; A v=0.26$ & $0.57-12.08 ; A v=3.89$ \\
\hline$\delta \mathrm{Eu}$ & $0.223-0.866 ; A v=0.55$ & 1-3.78; Av = 1.83 & $1.08-1.62 ; A v=1.15$ & $1.02-2.52 ; \mathrm{Av}=1.36$ & $0.385-1.49 ; A v=0.87$ & $0.8-4.27 ; A v=1.61$ \\
\hline$\delta \mathrm{Ce}$ & $0.53-2.11 ; A v=0.915$ & $0.54-2.01 ; A v=0.99$ & $0.43-1.52 ; \mathrm{Av}=1.03$ & $0.52-1.95 ; \mathrm{Av}=1.24$ & $0.64-1.5 ; A v=1.06$ & $0.73-3.92 ; A v=1.53$ \\
\hline
\end{tabular}


TABLE 3 | elationship table between the sequence of events and the chemistry of pyrite and chlorite.

\begin{tabular}{|c|c|c|c|c|c|c|c|}
\hline \multicolumn{2}{|l|}{ Age } & Permian & \multicolumn{5}{|c|}{ Carboniferous } \\
\hline \multicolumn{2}{|c|}{ Tectonic setting } & Post-collisional extension & \multicolumn{5}{|c|}{ Collision } \\
\hline \multicolumn{2}{|c|}{ Stage } & $\begin{array}{c}\text { Superimposed } \\
\text { transformation stage }\end{array}$ & \multicolumn{3}{|c|}{ Porphyry metallogenic stage } & \multicolumn{2}{|c|}{ Premineralization stage } \\
\hline \multirow{2}{*}{\multicolumn{2}{|c|}{ Mineral structure }} & $\begin{array}{l}\text { Chl-III (disseminated and } \\
\text { vein type with shear } \\
\text { deformation characteristics) }\end{array}$ & $\begin{array}{l}\text { Chl-II }{ }_{1} \text { (dense } \\
\text { disseminated and } \\
\text { stockwork type) }\end{array}$ & $\begin{array}{l}\mathrm{Chl}-\|_{2} \text { (disseminated } \\
\text { and vein type) }\end{array}$ & $\begin{array}{c}\text { Chl- } I_{3} \text { (mainly } \\
\text { disseminated type) }\end{array}$ & $\begin{array}{l}{\text { Chl- } i_{1} \text { (replacement }}_{\text {remnant, }} \\
\text { pseudomorphic) }\end{array}$ & $\begin{array}{c}\mathrm{Chl}_{-} \mathrm{i}_{2} \\
\text { (disseminated } \\
\text { type) }\end{array}$ \\
\hline & & $\begin{array}{c}\text { Py-III (disseminated and } \\
\text { vein type with fragmentized) }\end{array}$ & $\begin{array}{l}\text { Spotted (fine- } \\
\text { grained) }\end{array}$ & $\begin{array}{l}P y-\|_{1} \text { (disseminated } \\
\text { and vein type) }\end{array}$ & $\begin{array}{l}\mathrm{Py}-\mathrm{II}_{2} \text { (disseminated } \\
\text { and vein type) }\end{array}$ & \multicolumn{2}{|c|}{ Py-I (disseminated type) } \\
\hline \multirow{4}{*}{$\begin{array}{l}\text { Pyrite } \\
\text { (ppm) }\end{array}$} & $\mathrm{Co} / \mathrm{Ni}$ & $0.003-0.57 ; A v=0.16$ & - & $0.09-9.94 ; A v=2.71$ & $0.12-2.02 ; A v=0.53$ & \multicolumn{2}{|c|}{ 11.37-60.60; Av = 33.37} \\
\hline & $\mathrm{Co}+\mathrm{Ni}$ & $\begin{array}{c}313.88-6,523.06 \\
A v=3,098.99\end{array}$ & - & $\begin{array}{c}143.28-9,735.37 \\
A v=3,896.7\end{array}$ & $\begin{array}{c}16.92-1941.08 \\
A v=416.63\end{array}$ & \multicolumn{2}{|c|}{$\begin{array}{l}48.03-1714.26 \\
\text { Av }=584.66\end{array}$} \\
\hline & $\begin{array}{c}\text { Total } \\
\text { content }\end{array}$ & $\begin{array}{c}\text { 478.09-9,692.23 } \\
\text { Av }=4,351.3\end{array}$ & - & $\begin{array}{l}594-7,839.08 \\
A v=4,049.19\end{array}$ & $\begin{array}{c}125.64-2028.18 \\
A v=543.68\end{array}$ & \multicolumn{2}{|l|}{$\begin{array}{c}132.52-6,401.02 \\
A v=1982.41\end{array}$} \\
\hline & $\mathrm{Cu}$ & $8.95-50.68 ; A v=23.16$ & - & $\begin{array}{c}0.66-336.25 \\
A v=86.28\end{array}$ & $\begin{array}{l}2.96-43.6 \\
A v=16.38\end{array}$ & \multicolumn{2}{|l|}{$\begin{array}{c}5.94-4,133.8 \\
A v=950.33\end{array}$} \\
\hline \multirow[t]{6}{*}{$\begin{array}{l}\text { Chlorite } \\
\text { (ppm) }\end{array}$} & REE & $0.04-1.27 ; A v=0.49$ & $\begin{array}{l}0.056-0.77 \\
A v=0.425\end{array}$ & $0.02-3.42 ; A v=0.66$ & $\begin{array}{l}0.05-3.34 \\
A v=0.808\end{array}$ & $0.03-17.62 ; A v=1.8$ & $\begin{array}{l}0.02-0.71 \\
A v=0.248\end{array}$ \\
\hline & $\begin{array}{l}\text { LREE/ } \\
\text { HREE }\end{array}$ & $1.26-3.17 ; A v=1.75$ & $\begin{array}{l}0.07-0.59 \\
A v=0.296\end{array}$ & $0.11-1.19 ; A v=0.64$ & $0.08-0.85 ; A v=0.55$ & $0.12-5.95 ; A v=1.32$ & $\begin{array}{l}0.18-3.91 \\
A v=0.96\end{array}$ \\
\hline & $\delta \mathrm{Eu}$ & $0.8-4.27 ; A v=1.61$ & $1.08-1.62 ; A v=1.15$ & $1.02-2.52 ; A v=1.36$ & $\begin{array}{l}0.385-1.49 \\
A v=0.87\end{array}$ & $\begin{array}{c}0.223-0.866 \\
A v=0.55\end{array}$ & $\begin{array}{c}1-3.78 \\
A v=1.83\end{array}$ \\
\hline & $\delta \mathrm{Ce}$ & $0.73-3.92 ; A v=1.53$ & $0.43-1.52 ; A v=1.03$ & $0.52-1.95 ; A v=1.24$ & $0.64-1.5 ; A v=1.06$ & $0.53-2.11 ; A v=0.915$ & $\begin{array}{l}0.54-2.01 \\
A v=0.99\end{array}$ \\
\hline & $\mathrm{Ti}$ & $177.9-572.4 ; A v=299.68$ & $\begin{array}{c}11.07-176.09 \\
A v=37.54\end{array}$ & $\begin{array}{c}19-486.8 \\
A v=165.92\end{array}$ & $\begin{array}{c}21.64-1,055.86 \\
A v=334.43\end{array}$ & $\begin{array}{c}12.22-159.33 \\
A v=29.64\end{array}$ & $\begin{array}{c}10.96-573.20 \\
A v=71.45\end{array}$ \\
\hline & $\mathrm{Sr}$ & $0.71-2.02 ; A v=1.42$ & $1.83-7.1 ; A v=4.45$ & $\begin{array}{l}0.51-58.69 \\
A v=12.79\end{array}$ & $\begin{array}{c}0.37-121.19 \\
A v=17.18\end{array}$ & $\begin{array}{l}1.79-29.16 \\
A v=10.94\end{array}$ & $\begin{array}{l}0.86-7.49 \\
A v=4.79\end{array}$ \\
\hline
\end{tabular}

positive anomalies $(\delta \mathrm{Eu}=0.385-1.49)$, and negative $\mathrm{Ce}$ to positive anomalies $(\delta \mathrm{Ce}=0.64-1.5)$ in general. The results of $(\mathrm{La} / \mathrm{Sm}) \mathrm{n}(0.124-0.78)$ and $(\mathrm{Gd} / \mathrm{Yb}) \mathrm{n}(0.51-7.38)$ indicate that the internal fractionation of LREEs and HREEs is strong (Figure 9E).

The content of REEs in Chl-III ranges from 0.04 to $1.27 \mathrm{ppm}$, and the REE patterns are characterized by the relative enrichment of LREEs (LREE/HREE = 1.26-3.17), positive to weakly negative $\mathrm{Eu}$ anomalies $(\delta \mathrm{Eu}=0.80-4.27)$, and positive to weakly negative $\mathrm{Ce}$ anomalies $(\delta \mathrm{Ce}=0.73-3.92)$. The results of $(\mathrm{La} / \mathrm{Sm}) \mathrm{n}$ $(0.20-0.88)$ and $(\mathrm{Gd} / \mathrm{Yb}) \mathrm{n} \quad(1.80-8.08)$ indicate that the internal fractionation of LREEs and HREEs is strong (Figure 9F).

The in situ trace element analysis of chlorite shows that the trace element with the highest content in Chl-I $\sim$ Chl-III is Mn. The contents of $\mathrm{Ge}, \mathrm{Mo}, \mathrm{Cd}, \mathrm{In}, \mathrm{Sb}$, and REEs are usually less than $1 \mathrm{ppm}$, and $\mathrm{Mn}$ (348.55-3,466 ppm), Ni (80.73-477.43 ppm), Cu (0.01-388.38 ppm), and $\mathrm{Sr}(0.37-121.19 \mathrm{ppm})$ vary in large ranges. The contents of $\mathrm{V}, \mathrm{Ni}, \mathrm{Ti}$, and $\mathrm{Zn}$ mostly range from 100 to 500 ppm.

\section{DISCUSSION}

\section{Occurrence of Trace Elements in Pyrite}

The distribution of trace elements of pyrite is obviously various in the different mineralization stages in the Tuwu copper deposit. Three main occurrence forms of trace elements in pyrite and other sulfides are observed: 1) in the mineral lattice in the form of isomorphism, 2) in visible micron-scale inclusions of other minerals, and 3) as invisible nanometer-scale mineral particles (Leng, 2017). LA-ICP-MS can not only accurately obtain the content of in situ trace elements but also determine the occurrence forms of elements in minerals according to the spatial variation trend of elements with depth during LA (Zhou et al., 2010; Keith et al., 2016). The trend of each element in the LA-ICP-MS time resolution profile shows that the signals of $\mathrm{Sb}, \mathrm{Se}, \mathrm{Co}$, and $\mathrm{Ni}$ are generally stable and basically consistent with the signal distribution of $\mathrm{Fe}$, indicating that $\mathrm{Sb}, \mathrm{Se}$, $\mathrm{Co}$, and Ni may exist mainly in the form of isomorphism. Co and $\mathrm{Ni}$ replace $\mathrm{Fe}$ in pyrite, while $\mathrm{Se}$ and $\mathrm{Sb}$ can replace $\mathrm{S}$. The ablation signal curves of $\mathrm{Cu}, \mathrm{Pb}, \mathrm{Zn}, \mathrm{Cu}$, and $\mathrm{Au}$ are variable and irregular, indicating that these elements mainly exist in the form of microinclusions in pyrite (Figure 8).

Moreover, most curves of $\mathrm{Cu}, \mathrm{Pb}$, and $\mathrm{Ag}$ appeared in local peaks (Figure 8), which indicates that $\mathrm{Cu}, \mathrm{Pb}$, and $\mathrm{Ag}$ elements mainly exist in pyrite in the form of microinclusions (chalcopyrite, galena, and native silver). In the correlation diagram (Figure 10), $\mathrm{Cu}$ shows a significant positive correlation with $\mathrm{Pb}$ and $\mathrm{Ag}$. The degree of correlation was better in $\mathrm{Py}-\mathrm{II}_{1}$ and $\mathrm{Py}-\mathrm{II}_{2}$ than in $\mathrm{Py}-\mathrm{I}$ and $\mathrm{Py}-\mathrm{III}$ because $\mathrm{Pb}$ and $\mathrm{Ag}$ replacements of $\mathrm{Fe}$ are difficult by isomorphism, while $\mathrm{Cu}$ is known to occur as a substitution for Fe in many Fe-bearing sulfides (i.e., pyrite and pyrrhotite) and silicates (i.e., biotite and hornblende), indicating that more $\mathrm{Cu}$ exists in the form of 

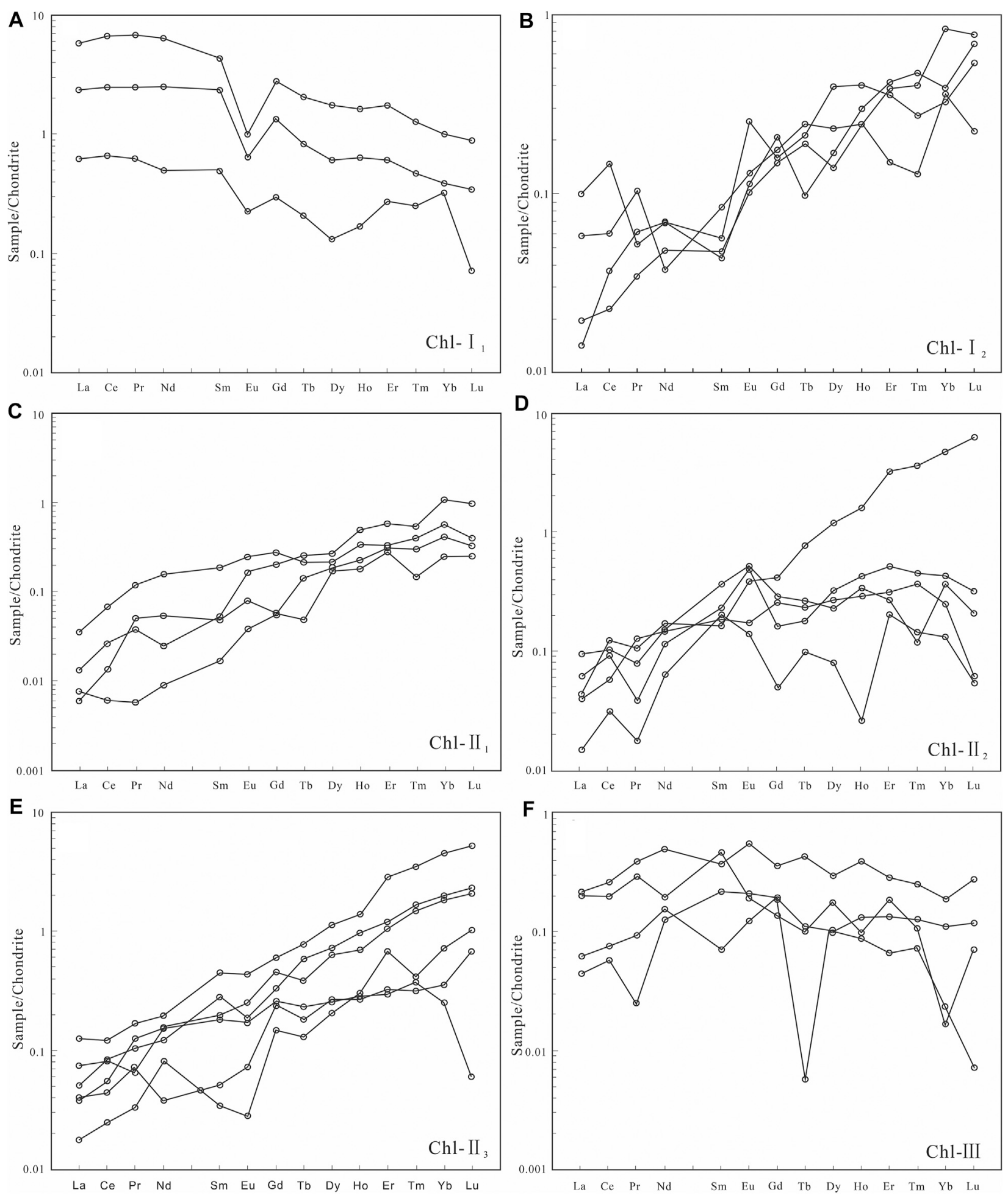

FIGURE 9 |Chondrite-normalized rare earth element patterns of chlorite from different mineralization stages of the Tuwu porphyry Cu deposit in eastern Tianshan, Xinjiang, NW China (normalization values from Talor and McLennan, 1985).

isomorphism in Py-I and Py-III than in Py- $\mathrm{II}_{1}$ and $\mathrm{Py}-\mathrm{II}_{2}$. A weak positive correlation occurs between $\mathrm{Cu}$ and As in pyrite. The signal distribution of As is consistent with that of Fe. A local peak of As appears in a small number of samples and corresponds to the high value point of $\mathrm{Cu}$, which indicates that As not only exists in arsenopyrite but also replaces $\mathrm{S}$ in pyrite via isomorphism. The 

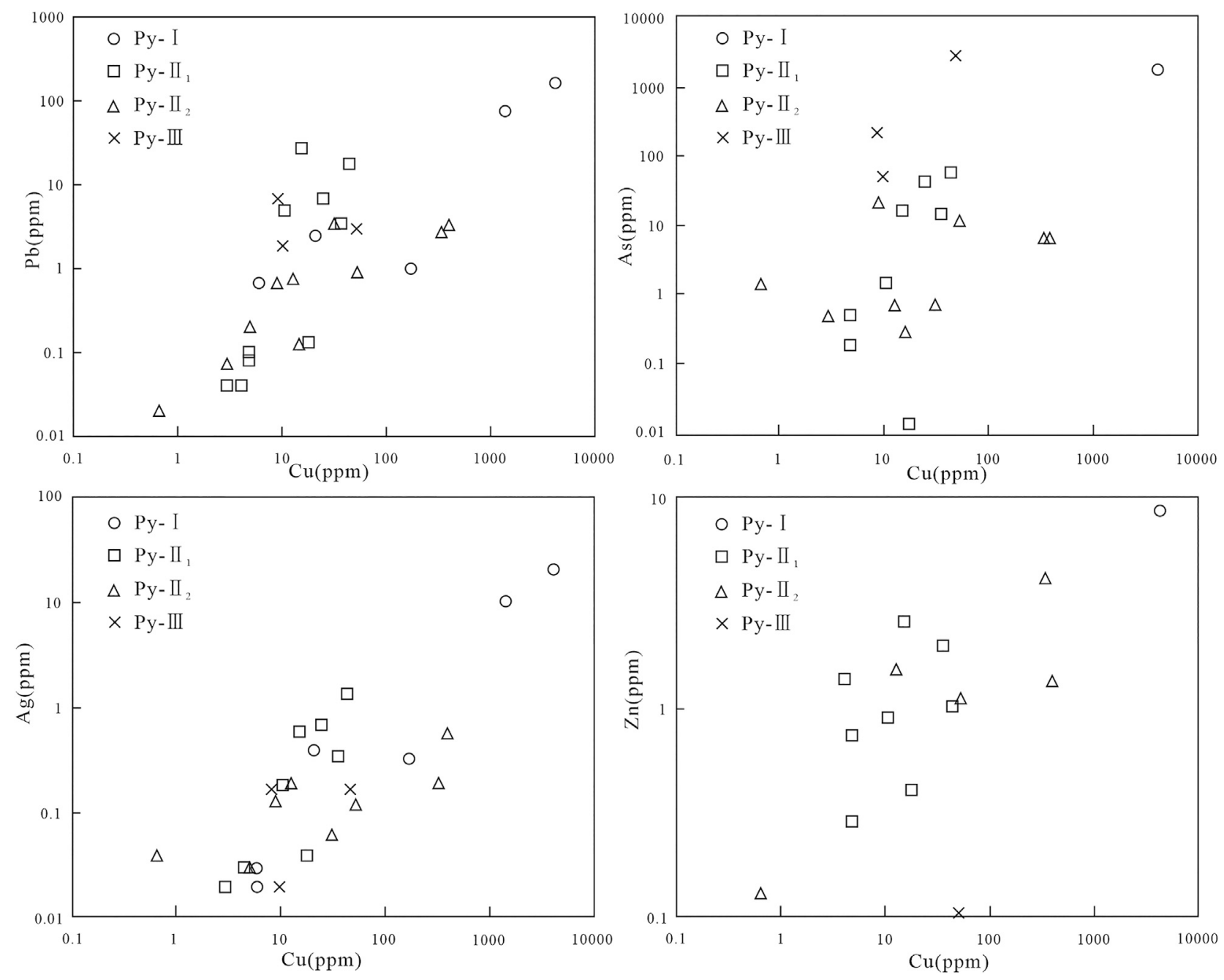

FIGURE 10 | Correlation diagrams for the trace elements of pyrite from the Tuwu porphyry Cu deposit in eastern Tianshan, Xinjiang, NW China.

mass fraction of As fluctuates greatly, with obvious high values in Py-I and Py-III. Above all, these results indicate that the content of As was greatly affected by fluid in later stages, and its content may have a certain effect on the precipitation and occurrence of $\mathrm{Cu}$. Zinc appeared in local peaks (Figure 8), which indicates that $\mathrm{Zn}$ may exist in the form of sphalerite microinclusions in pyrite, and the content of $\mathrm{Zn}$ appears to be highly variable in Py-III and Py-III. Moreover, considering that the ionic radius of $\mathrm{Zn}^{2+}$ is $0.74 \AA$ and $\mathrm{Fe}^{2+}$ is $0.76 \AA$, $\mathrm{Zn}$ may replace $\mathrm{Fe}$ in the pyrite lattice via isomorphism.

\section{Genesis of Pyrite}

Trace elements entering into the pyrite lattice in solid solution form are restricted by the chemical composition of the surrounding rock, mineral assemblage, and fluid properties. Because different hydrothermal fluids usually form under different physicochemical conditions, the contents of trace elements in pyrite vary significantly. $\mathrm{Co}$ and $\mathrm{Ni}$ are the most common trace elements in pyrite, and their contents and the $\mathrm{Co} / \mathrm{Ni}$ ratio are often used to trace the genesis, deposition environment, and formation temperature of pyrite (Bralia et al., 1979; Campbell and Ethier, 1984; Bajwah et al., 1987; Cook, 1996). Different genetic types of pyrite usually have different $\mathrm{Co} / \mathrm{Ni}$ ratios (Bralia et al., 1979; Brill, 1989; Large et al., 2011). The $\mathrm{Co} / \mathrm{Ni}$ ratio of synsedimentary pyrite is usually less than 1 , whereas the $\mathrm{Co} / \mathrm{Ni}$ ratio of hydrothermal pyrite varies widely but is generally greater than 1 ; in particular, the $\mathrm{Co} / \mathrm{Ni}$ ratio of volcanic hydrothermal pyrite is generally between 5 and 100 (Bralia et al., 1979; Craig et al., 1998; Franchini et al., 2015; Keith et al., 2016) because of the difference in the octahedral site preference energies of $\mathrm{Co}(7.4 \mathrm{cal} / \mathrm{mol})$ and $\mathrm{Ni}$ (20.6 cal/mol) (Bralia et al., 1979). With the process of magma crystallization differentiation, $\mathrm{Ni}$ prefers to enter minerals with a high octahedral ratio (such as olivine and orthopyroxene) during early crystallization, while Co becomes relatively enriched in minerals forming in later-stage crystallization, thus causing the $\mathrm{Co} / \mathrm{Ni}$ ratio of magmatic and hydrothermal pyrite to be greater than 1 . Therefore, the $\mathrm{Co} / \mathrm{Ni}$ ratio of pyrite that precipitated in response to magmatic or 


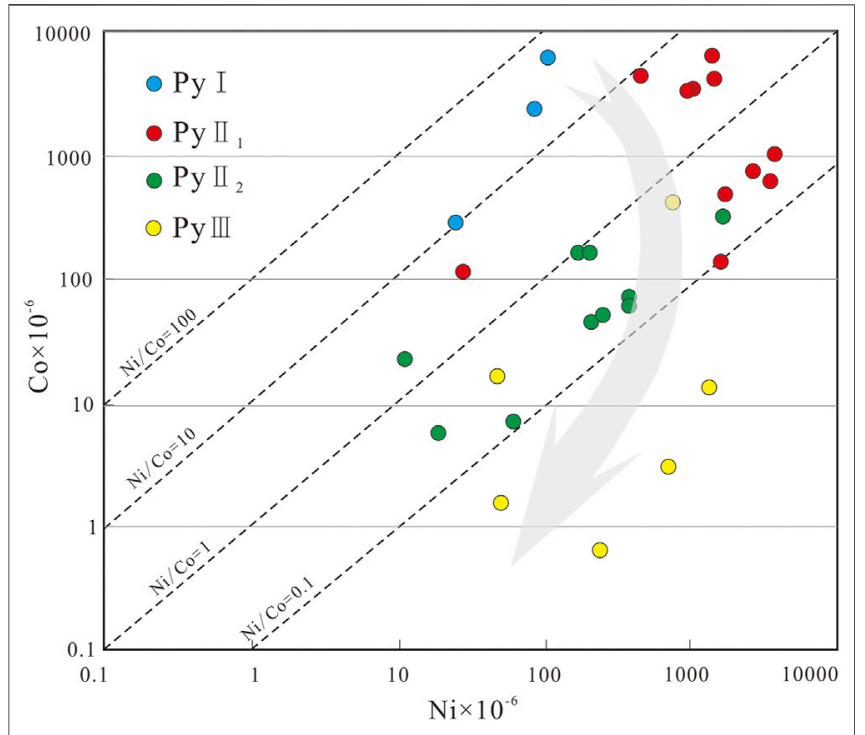

FIGURE 11 | Co-Ni genetic diagram for the different types of pyrite from the Tuwu porphyry Cu deposit in eastern Tianshan, Xinjiang, NW China.

hydrothermal activity is generally greater than 1 (Song and Zhang, 1986; Zhou et al., 2010; Leng, 2017).

The test results show that the Co and $\mathrm{Ni}$ contents of pyrite in the Tuwu mining area vary greatly and tend to decrease from PyI, Py- $\mathrm{II}_{1}$, and $\mathrm{Py}-\mathrm{II}_{2}$ to Py-III, which reflects the fluid evolution characteristics of the Tuwu mining area to a certain (Figure 11). The $\mathrm{Co} / \mathrm{Ni}$ ratio of the pyrite in the weakly propylitized zone (PyI) ranges from 11.37 to 60.60 . The siderophile elements $\mathrm{Co}$ and $\mathrm{Ni}$ are often enriched in basic or ultrabasic rocks. Thus, the anomalous enrichment of $\mathrm{Co}$ and $\mathrm{Ni}$ elements and high $\mathrm{Co} / \mathrm{Ni}$ ratios in Py-I reflect a magmatic origin and are closely related to the formation of the CQ group volcanic rocks.

The $\mathrm{Co} / \mathrm{Ni}$ ratio of the pyrite in the pyritization, sericitization, and silicification zone $\left(\mathrm{Py}-\mathrm{II}_{1}\right)$ ranges from 0.09 to 9.94 and is generally consistent with the characteristics of magmatic or hydrothermal pyrite. However, the lower the $\mathrm{Co} / \mathrm{Ni}$ ratio of $\mathrm{Py}-\mathrm{II}_{1}$ is, the more the fragmentized pyrite is. Some studies have shown that using the $\mathrm{Co} /$ $\mathrm{Ni}$ ratio as a criterion to distinguish the boundary of different types of ore deposits is not reliable, especially for hydrothermal deposits (Song and Zhang, 1986; Wang, 1989). Geochemistry characteristics of fluid inclusions and $\mathrm{H}, \mathrm{O}$, and $\mathrm{S}$ isotopes show that the ore-forming fluid mainly evolved from magmatic hydrothermal fluids, meteoric water, and metamorphic fluid (Liu et al., 2009; Wang et al., 2018). Therefore, $\mathrm{Py}-\mathrm{II}_{1}$ may have been affected by the addition of meteoric water or other fluids in the late stage of mineralization, and the $\mathrm{Co} / \mathrm{Ni}$ ratio of the pyrite shows certain characteristics of sedimentary origin. $\mathrm{Py}-\mathrm{II}_{1}$ is depleted in low-temperature elements such as As, Se, and Te, which is consistent with the geochemical characteristics of high-temperature hydrothermal pyrite.

The Co/Ni ratio of the pyrite in the sericitization zone $\left(\mathrm{Py}-\mathrm{II}_{2}\right)$ ranges from 0.12 to 2.02 (average 0.53 ), and the corresponding data points mainly plot in the field of sedimentary-origin pyrite. The characteristics of trace elements in $\mathrm{Py}-\mathrm{II}_{2}$ indicate the complexity of pyrite genesis. The formation of this pyrite may have involved the mixing of magmatic hydrothermal fluid and meteoric water. The meteoric water erased most of the magmatichydrothermal information. In addition, calcite, a typical lowtemperature mineral, is abundant in this stage.

The $\mathrm{Co} / \mathrm{Ni}$ ratio of the pyrite in the chloritization-sericitization zone (Py-III) ranges from 0.003 to 0.57 . Cobalt and $\mathrm{Ni}$ in pyrite can form $\mathrm{CoS}_{2}$ and $\mathrm{NiS}_{2}$ by replacing Fe via isomorphism. The unit cell parameters of pyrite are lengthening because the bond lengths of Co-S and $\mathrm{Ni}-\mathrm{S}$ are greater than that of $\mathrm{Fe}-\mathrm{S}$. Pyrite can form a continuous solid solution series of $\mathrm{FeS}_{2}-\mathrm{CoS}_{2}$ and a discontinuous solid solution series of $\mathrm{FeS}_{2}-\mathrm{NiS}_{2}$ (Bralia et al., 1979; Song and Zhang, 1986; Craig et al., 1998). The content of $\mathrm{FeS}_{2}-\mathrm{CoS}_{2}$ is 2-3 orders of magnitude lower than that of $\mathrm{FeS}_{2}-\mathrm{NiS}_{2}$ in Py-III. The rocks in the chloritization-sericitization zone are characterized by shear deformation, which manifests as highly schistose local ore bodies and significant elongation of minerals. Overall, the formation of pyrite in this stage appears to involve superimposed transformation by metamorphic hydrothermal fluids, which is consistent with the geochemistry of fluid inclusions and isotopes (Liu et al., 2009; Wang et al., 2018).

The temperature controls the types and contents of trace elements in pyrite. Generally, a higher temperature of formation corresponds to more trace element types in pyrite and a higher content of trace elements (Craig et al., 1998; Large et al., 2007; Keith et al., 2016). Pyrite with different total trace element contents can be divided into three different stages. Comprehensive research on the contents of different trace elements and the $\mathrm{Co} / \mathrm{Ni}$ ratio in pyrite shows that the total trace element content is highest in Py-I (4,351.34 ppm), which mainly formed in the later period of volcanic activity. The high copper content may reflect the high copper background value of the volcanic rocks in the CQ Group.

The total trace element contents in $\mathrm{PyII}_{1}$ and $\mathrm{PyII}_{2}$ are 4,049.19 and $543.68 \mathrm{ppm}$, respectively. Combined with previous studies of fluid inclusions (Liu et al., 2009; Han et al., 2010; Wang et al., 2018), these results reflect the variation characteristics of trace elements in pyrite from the main mineralizing stage to the late stage of the porphyry mineralization period. The total trace element content in Py-III is $1982.41 \mathrm{ppm}$. The temperature of the metamorphic hydrothermal fluid is between $150^{\circ} \mathrm{C}$ and $300^{\circ} \mathrm{C}$, and the copper is obviously enriched by the superimposed transformation of the metamorphic hydrothermal solution. This finding explains why the temperature of the main metallogenic stage in the Tuwu copper deposit was approximately $200^{\circ} \mathrm{C}$ (Liu et al., 2009; Han et al., 2010), which is mainly because earlier high-temperature fluid inclusions were superimposed by ductile shearing.

\section{Rare Earth Element Geochemistry of Chlorite}

The geochemical similarity of REEs allows them to be used in tracing applications, which is similar to isotopic values for the process of mineralization (Michard and Albarède, 1986; Maclean, 1988; Fulignati et al., 1999). By studying the REE composition characteristics of minerals from different metallogenic stages, we can determine the geochemical behavior of REEs during the processes of ore formation and alteration (Maclean, 1988; 
Chen and Fu, 1991, 1997). Such work can effectively constrain the ore-forming material source, the metallogenic physicochemical environment, the fluid evolution process, and ore genesis (Chen and Fu, 1991; Bortnikov et al., 2008; Parsapoor et al., 2009; Tan et al., 2016, 2018). Research has shown that the geochemical behavior of REEs in minerals is mainly related to the secondary minerals formed by alteration, the content of REEs in hydrothermal fluids, the formation temperature of minerals, the $\mathrm{pH}$ and $\mathrm{Eh}$ of hydrothermal systems, the crystal chemical properties of minerals, and the partition coefficient of REEs between minerals and fluids (Michard and Albarède, 1986; Michard, 1989; Bortnikov et al., 2008). Previous studies on the REE characteristics of argillization zones (Karakaya et al., 2012), silicification zones (Terakado and Fujitani, 1998; Khalili and Malekmahmoudi, 2012), sericitization-silicification zones (Parsapoor et al., 2009), propylitization zones (Fulignati et al., 1999), and potassic zones (Taylor and Mclennan, 1985) show that the contents of REEs differ among different alteration zones.

\section{Rare Earth Element Characteristics of Chlorite in the Premineralization Period}

Microstructure research has shown that there are two main formation mechanisms of chlorite in the Tuwu copper deposit: 1. metasomatic crystallization, which is chlorite formation via the metasomatism of biotite and hornblende, and in those rocks by late-stage hydrothermal fluids, 2. precipitation crystallization, which is the chlorite precipitated from hydrothermal fluids rich in $\mathrm{Fe}$ and $\mathrm{Mg}$ in the cracks or gaps between minerals. Metasomatic crystallization chlorite $\left(\mathrm{Chl}-\mathrm{I}_{1}\right)$ in the weakly propylitized zone mainly formed by late hydrothermal alteration of hornblende, pyroxene, and biotite in basalt. This chlorite generally inherited the REE distribution pattern of the volcanic rocks in the CQ group in the Tuwu mining area (Hou et al., 2005, 2006; Zhao et al., 2014). The precipitation crystallization chlorite (Chl- $\left.\mathrm{I}_{2}\right)$ is mainly associated with quartz, epidote, and minor pyrite. The REE patterns of Chl- $\mathrm{I}_{2}$ are characterized by the relative enrichment of LREEs and positive $\mathrm{Eu}$ anomalies, which may be due to the $\mathrm{Eu}$ in minerals that were in equilibrium with the hydrothermal solution entering the fluid in the form of $\mathrm{Eu}^{2+}$ during the rock-water interaction and separated from the other REEs. As a result, Chl- $\mathrm{I}_{1}$ has stronger negative $\mathrm{Eu}$ anomalies than the volcanic rocks. In contrast, Eu is enriched in the fluid, and Chl- $\mathrm{I}_{2}$, which precipitated from the fluid, has positive Eu anomalies.

The enrichment degree of $\mathrm{Eu}$ in the fluid is closely related to temperature. Even in relatively oxidized environments, $\mathrm{Eu}$ in hydrothermal solutions mainly exists in the form of $\mathrm{Eu}^{2+}$ at relatively high temperatures (Bortnikov et al., 2008; Parsapoor et al., 2009; Fadda et al., 2012). Therefore, Chl- $\mathrm{I}_{2}$ has a high crystallization temperature, and the total content of REEs in Chl$\mathrm{I}_{1}$ is generally 1-2 orders of magnitude higher than those in Chl-II and Chl-III, indicating the characteristics of the fluids related to Chl-I were obviously different from those of the fluids in the metallogenic epoch. The fluid likely was a volcanogenic hydrothermal solution that formed during late volcanic activity. The content of $\mathrm{Cu}$ in chlorite is generally high. The closer to the ore body, the higher the $\mathrm{Cu}$ content. This relationship reflects that the volcanic rocks in the CQ group have a high background value of $\mathrm{Cu}$. In addition, the volcanic syngenetic hydrothermal activity primarily led to the enrichment in copper.

\section{Rare Earth Element Characteristics of Chlorite in Porphyry Metallogenic Stage}

Similar LREE enrichment is observed in the REE distribution patterns of Chl- $\mathrm{II}_{1}$, which is associated with epidote, quartz, calcite, and zoisite in the strong propylitization zone. Chl- $\mathrm{II}_{2}$ is associated with sericite, pyrite, and chalcopyrite in the pyritization-sericitization-silicification zone, and $\mathrm{Chl}_{-} \mathrm{II}_{3}$ is associated with sericite, calcite, pyrite, and chalcopyrite in the sericitization-silicification zone. In addition, the total REE content in Chl-II is significantly lower than that of Chl-I and the $\mathrm{Eu}$ anomalies in $\mathrm{Chl}-\mathrm{II}_{1}, \mathrm{Chl}-\mathrm{II}_{2}$, and $\mathrm{Chl}-\mathrm{II}_{3}$ range from positive to negative. The reasons for the above REE characteristics in Chl-II are as follows.

1) Because chloritization occurs in neutral to alkaline $\mathrm{pH}$ conditions with relatively low temperatures $\left(<300^{\circ} \mathrm{C}\right)$ (Reed, 1997), the REEs in the fluid mainly form complexes with $\mathrm{SO}_{4}{ }^{2-}$ and $\mathrm{CO}_{3}{ }^{2-}$. While $\mathrm{SO}_{4}{ }^{2-}$ does not preferentially bind with REEs, $\mathrm{CO}_{3}{ }^{2-}$ does preferentially bind with REEs to form complexes (Foster, 1962; Bortnikov et al., 2008; Inoue et al., 2010). The secondary minerals formed from this fluid are relatively rich in heavy REEs.

LREEs have a larger ionic radius and smaller atomic weight than HREEs; therefore, LREEs are more easily leached from minerals (Bortnikov et al., 2008; Ye et al., 2011; Karakaya et al., 2012). The REE patterns of Chl-II are characterized by the relative enrichment of HREEs, indicating that it experienced leaching action of late hydrothermal fluids. Additionally, the similarity in REE distribution patterns indicates that $\mathrm{Chl}-\mathrm{II}_{1}$, $\mathrm{Chl}-\mathrm{II}_{2}$, and $\mathrm{Chl}-\mathrm{II}_{3}$ have the same material source and geochemical behavior.

2) Although accessory minerals exist in only very small quantities in most rocks, the REE distribution coefficients in accessory minerals are very high; therefore, they play a great role in controlling the REE content in rocks. The content of REEs in plagiogranite porphyry $(24.87-55.88 \mathrm{ppm})$ is lower than that in volcanic rock (50.64-117.73 ppm) (Wang et al., 2014, 2015; Zhao et al., 2014), and the accessory minerals in plagiogranite porphyry are more than that in the volcanic rock, which causes the content of REEs in Chl-II to be lower than that in Chl-I.

3) The stability of $\mathrm{Eu}^{2+}$ is controlled not only by the temperature, $\mathrm{pH}$, Eh, and oxygen fugacity of the open system but also by the supply of alteration minerals. Previous studies have found that $\mathrm{Eu}^{3+}$ tends to occur under low $\mathrm{pH}$ and high oxygen fugacity (Sverjensky, 1984; Bau, 1991; Hedenquist, 1995). The valence state of Eu will transform from $\mathrm{Eu}^{3+}$ to $\mathrm{Eu}^{2+}$ and fractionate from other $\mathrm{REEs}^{3+}$ in a reducing environment, and $\mathrm{Eu}^{2+}$ will remain in a stable state under a low oxygen fugacity environment. The $\mathrm{Chl}^{-\mathrm{II}_{1}}$ in the strong propylitization zone and the $\mathrm{Chl}_{-} \mathrm{II}_{2}$ in the 
sericitization-silicification zone exhibit positive $\mathrm{Eu}$ anomalies, indicating that the hydrothermal system featured a higher temperature $\left(>250^{\circ} \mathrm{C}\right)$, a low $\mathrm{pH}$, and relatively reducing conditions and the chlorite generally inherited the REE distribution pattern of the ore-forming fluid. The stronger positive $\mathrm{Eu}$ anomalies in $\mathrm{Chl}-\mathrm{II}_{2}$ may is consistent with the large amount of sulfide that crystallized from the ore-forming fluid. With the development of mineralization and the addition of meteoric water, the metallogenic environment will transform from a relatively reducing environment to a relatively oxidizing environment. The occurrence of a large number of lowtemperature minerals, such as calcite, indicates that the mineralization temperature has obviously decreased. $\mathrm{Chl}-\mathrm{II}_{3}$ formed at this stage shows obviously negative $\mathrm{Eu}$ anomalies. $\mathrm{Eu}^{2+}$ has an ionic radius similar to that of $\mathrm{Ca}^{2+}$ and often occurs in calcite via isomorphism. Therefore, a large amount of calcite precipitation also reduces the content of $\mathrm{Eu}$ in the oreforming fluid.

\section{Rare Earth Element Characteristics of Chlorite in the Superimposed Transformation Period}

The chlorite in the rich ore bodies (Chl-III) is characterized by obvious shear deformation characteristics. The REE distribution pattern exhibits LREE enrichment, and the fractionation of HREEs and LREEs is not obvious. This result suggests that the REE distribution pattern of Chl-III is apparently different from that of Chl-I and Chl-II. The formation depth of the Tuwu copper deposit is approximately $2-3 \mathrm{~km}$ (Wang et al., 2018). Combined with the significant deformation characteristics of the minerals in the chloritization-sericitization zone, we can infer that the Tuwu copper deposit was located in the brittle-ductile transitional zone. As the water/rock ratio increased, the diffusion pattern of the fluids shifted from percolation to infilling. The REE distribution pattern of Chl-III was mainly controlled by the fluid properties. In addition, the fluid source is also an important factor in determining the composition of REEs. The REE distribution patterns of the plagiogranite porphyry and dioritic porphyrite are generally consistent with that of Chl-III. However, the fractionation of HREEs and LREEs in Chl-III is obviously low, which indicates that the physical and chemical conditions of the Chl-III formation are apparently different from those of Chl-I and Chl-II. The positive $\mathrm{Eu}$ anomalies of Chl-III may indicate that Chl-III formed in a relatively reducing environment at relatively high temperatures.

\section{Spatial Variation Pattern of Trace Elements in Chlorite}

In the formation process of porphyry deposits, a large alteration halo usually occurs around the center of the ore body. In this alteration halo, the mineral association and chemical composition may show certain zonation (Lowell and Guilbert, 1970). The classical metallogenic model of porphyry copper provides theoretical support for exploring porphyry deposits on the regional scale. However, ore bodies of economic value tend to be concentrated in the range of $1 \mathrm{~km}$, and there are many alterations that are irrelevant to the ore-forming process (Inoue et al., 2010; Fadda et al., 2012). Therefore, effective identification of metallogenic and nonmetallogenic alteration systems is of great significance for the exploration of porphyry deposits. In recent years, the geochemical characteristics of altered minerals have attracted the widespread attention (Inoue et al., 2010; Cooke et al., 2014; Wilkinson et al., 2015; Xiao et al., 2018). Understanding these characteristics not only helps reveal the genesis of ore deposits but also provides technical guidance for prospecting exploration. Currently, based on electron probe and LA-ICP-MS analyses of altered minerals in porphyry systems, scholars have documented spatial variations in trace elements in altered minerals (Cooke et al., 2014; Wilkinson et al., 2015).

These achievements have been applied and verified many times in the field of exploration in China and elsewhere. They are commonly used to assist exploration. The systematic electron probe and LA-ICP-MS analysis of chlorite in the propylitization zone of the Batu Hijau superlarge porphyry copper (gold) deposit in Indonesia has shown that $\mathrm{K}, \mathrm{Li}, \mathrm{Mg}, \mathrm{Ca}, \mathrm{Sr}, \mathrm{Ba}, \mathrm{Ti}, \mathrm{V}, \mathrm{Mn}, \mathrm{Co}$, $\mathrm{Ni}, \mathrm{Zn}$, and $\mathrm{Pb}$ can enter the crystal lattice of chlorite. With distance from the ore body, the contents of $\mathrm{Ti}, \mathrm{V}$, and $\mathrm{Mg}$ decrease exponentially, while the contents of $\mathrm{K}, \mathrm{Li}, \mathrm{Ca}, \mathrm{Sr}, \mathrm{Ba}$, $\mathrm{Mn}, \mathrm{Co}, \mathrm{Ni}, \mathrm{Zn}$, and $\mathrm{Pb}$ increase exponentially (Cooke et al., 2014; Wilkinson et al., 2015; Xiao et al., 2018).

The spatial variations in the trace element compositions in chlorite in the Tuwu mining area are similar to those in the Batu Hijau superlarge porphyry copper (gold) deposit. For example, the contents of high field strength elements, such as Ti and V, are higher in Chl-II and Chl-III near the ore body than in Chl-I far from the ore body. The degree of isomorphism of $\mathrm{Ti}$ in chlorite is mainly controlled by the temperature of mineral crystallization (Wilkinson et al., 2015). Therefore, the spatial variation in $\mathrm{Ti}$ content in chlorite also provides information on the thermal anomaly around the mineralization center. The variation range of Ti contents in Chl-II and Chl-III can reach 2-3 orders of magnitude within $300 \mathrm{~m}$. However, the migration ability of high field strength elements, such as $\mathrm{Ti}$ and $\mathrm{V}$, in hydrothermal fluid is quite limited. The significant differences in $\mathrm{Ti}$ content in chlorite in the porphyry mineralization stage in the Tuwu mining area indicate that the migration and diffusion of $\mathrm{Ti}$ were affected not only by temperature but also by the repeated action of hydrothermal. Moreover, the Ti contents in Chl-I vary little within a range of $1 \mathrm{~km}$, which suggests that Chl-I in the volcanic rocks mainly formed in the volcanism and was scarcely affected by late hydrothermal activity.

With increasing distance from the ore body, the contents of $\mathrm{K}$, $\mathrm{Ca}, \mathrm{Sr}, \mathrm{Ba}$, and other large ion lithophile elements decrease but the degree of spatial variation is not as obvious as in a previous study (Wilkinson et al., 2015). This finding shows that the large ion lithophile elements migrated more easily in the late-stage hydrothermal solution. The content ranges of $\mathrm{K}, \mathrm{Ca}, \mathrm{Sr}$, and $\mathrm{Ba}$ in Chl-I are obviously less than those in Chl-II and Chl-III, which suggests that Chl-II and Chl-III were affected by multiple phases of hydrothermal activity. The contents of $\mathrm{Mn}$ and $\mathrm{Zn}$ in chlorite gradually increase with distance from the center of the ore body, with a maximum value at a distance of approximately $1.2 \mathrm{~km}$; beyond this distance, they gradually decrease until they stabilize. This pattern reflects the horizontal movement of Mn and $\mathrm{Zn}$ with the magmatic hydrothermal fluid in the surrounding 

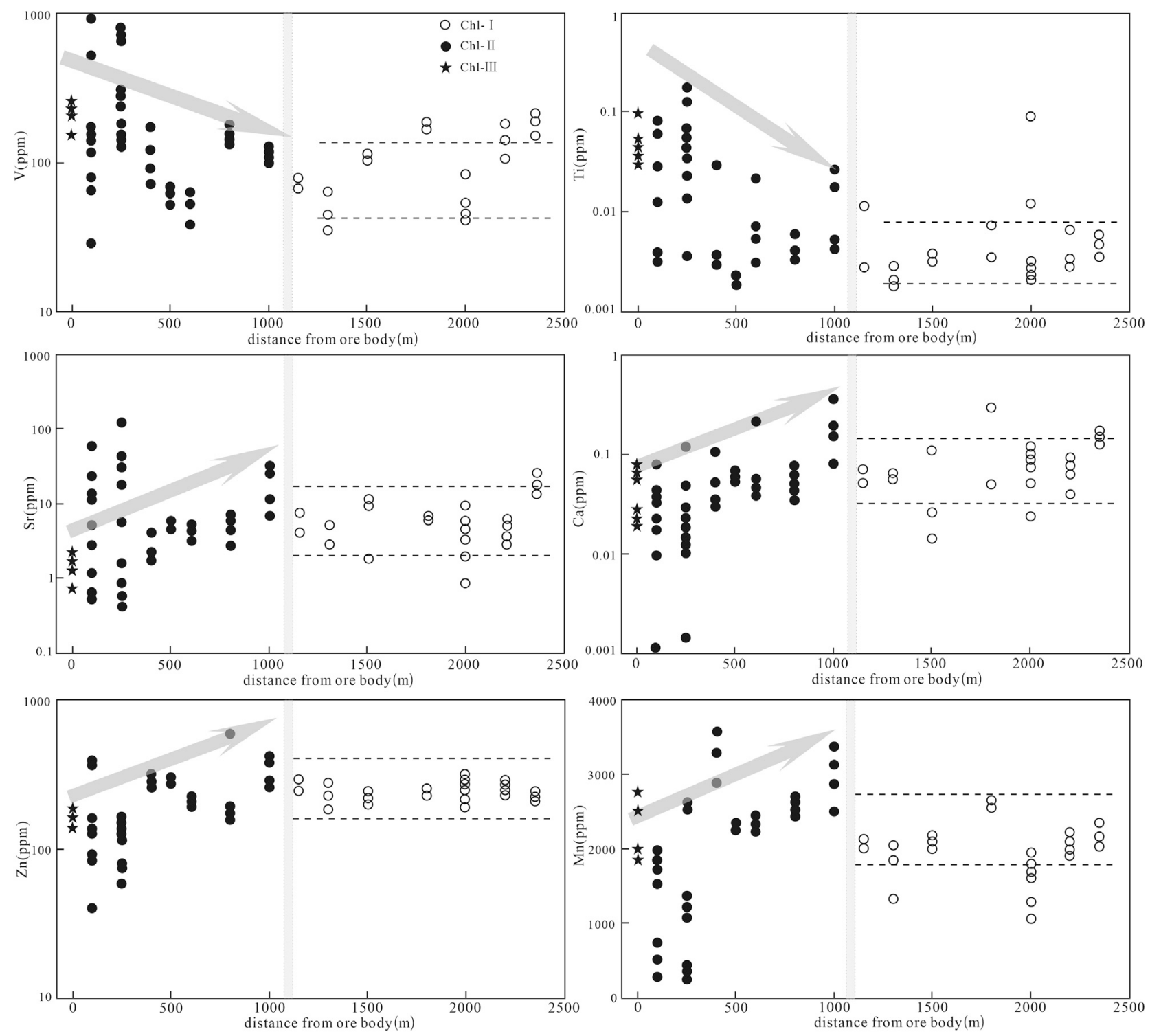

FIGURE 12 | Trace element spatial variations in chlorite for different alteration zones of the Tuwu porphyry Cu deposit in eastern Tianshan, Xinjiang, NW China.

rock. The high contents of $\mathrm{Mn}$ and $\mathrm{Zn}$ in Chl-I reflect the high background values of $\mathrm{Mn}$ and $\mathrm{Zn}$ in the volcanic rocks in the CQ group (Figure 12).

\section{Influence of Ductile Shearing on the Tuwu Deposit}

Although the Tuwu deposit experienced intense superimposed reformation, there are many features and characteristics that match those of a porphyry system. 1) Although most of the $\mathrm{Cu}$ in the Tuwu deposit is hosted in the surrounding rock, the ore-bearing plagiogranite porphyries are almost wholly mineralized. 2) Although the shape of the orebodies is mainly tabular, the ore structures are disseminated and veinletdisseminated and form stockwork. 3) Although the boundaries of the alteration zone are not obvious, the concentric alteration zones from the interior outward in succession are the potassic zone, the quartz-sericite zone, and the propylitic zone in general.

Compared with young porphyry systems, ancient porphyry systems tend to be subjected to later superimposed transformations. However, few studies have focused on the deformation and mineralization enrichment of porphyry deposits. The following evidence is presented in this article to demonstrate that the Tuwu porphyry deposit has undergone strong deformation and transformation. 1) The Tuwu porphyry deposit has experienced regional ductile shear activity after mineralization. The mining area is located in the northern influence zone of the large QiugemingtashiHuangshan ductile shear zone. 2) The intensity of the ductile deformation zone in the Tuwu-Yandong mining area is stronger in the south and weaker in the north. The southern part features a 
continuous strong ductile deformation zone and the northern part features a weak ductile deformation zone composed of several discretely distributed small lenticular and zonal ductile deformation zones. The ore has mylonite, augen, and fragmented structures. The mylonitic foliation developed in the ore body provides the main fine structural space for ore minerals ( $\mathrm{Ma}$ et al., 2002, 2003). These characteristics indicate that the Tuwu mining area formed in the brittle-ductile transition zone. 3) Considerable research has already been done on fluid inclusions in the Tuwu copper deposit. Previous studies have found that the temperatures of the main ore-forming periods are approximately $200^{\circ} \mathrm{C}$ (Liu et al., 2009; Han et al., 2010), while others found that the temperatures are approximately $350^{\circ} \mathrm{C}$ (Wang et al., 2015). This discrepancy may be related to the different fluid properties of ore bodies in different positions. 4) Oriented secondary fluid inclusions are well developed in the deformation zone (Liu et al., 2009; Wang et al., 2018), indicating that the shape of the orebody changes from a disseminated low-grade to a vein highgrade orebody by tectonism. 5) The Tuwu mining area can be divided into three tectonic facies according to the characteristics of deformation. The ductile compression zone belongs to the moderately deep ductile deformation facies; the brittle-ductile shear zone belongs to the moderately shallow transition facies between ductile and brittle deformation, and the brittle fault zone belongs to the shallow structural facies. The fact that the moderately deep, moderately shallow, and shallow structures appear on the same plane indicates that they did not form at the same time but are instead the result of superimposing different structures in ore-bearing geologic bodies that have been uplifted several times and that have undergone multiple phases of structural deformation at different depths ( $\mathrm{Ma}$ et al., 2003). 6) Closer to the ductile deformation zone, the contents of copper, sericite, chlorite, and quartz increase, and the oriented arrangement characteristics of these mineral become more obvious. There are some chalcopyrite and pyrite distributed along foliations of mylonite in the ductile deformation zone, and the chalcopyrite veins filled the fractures in the pyrite. These characteristics indicate that the ore has experienced strong deformation and the copper remobilized. 7) In situ trace element analysis of chlorite and pyrite in different stages shows distinctly different geochemical compositions Table 3.

The mineralization of the Tuwu and Yandong copper deposits occurred in the early Carboniferous at the southern belt of Dananhu-Haerlik island arc, and these deposits are separated by only $5 \mathrm{~km}$. The ore-forming bodies are all plagiogranite porphyry and have similar mineralization processes (Chen et al., 2005; $\mathrm{Hu}$ et al., 2011; Shen et al., 2012; Pan et al., 2013). However, $80 \%$ of the $\mathrm{Cu}$ in the Tuwu mining area is hosted in the diorite porphyry, while $90 \%$ of the $\mathrm{Cu}$ in the Yandong mining area is hosted in the plagiogranite porphyry (Hu et al., 2011). The superposition and transformation of late ductile shearing on the two deposits in different spatial positions may be one of the reasons for this difference. The plagiogranite porphyry brought metallogenic materials and formed the initial mineralization of the Tuwu copper deposit, while the late ductile shear process accompanied by strong hydrothermal activity resulted in the activation and enrichment of copper in the mining area. Moreover, the morphology of the ore bodies is mainly tabular, which is quite different from that of typical porphyry deposits.

These findings may be related to changes in the tectonic environment of the Tuwu mining area caused by ductile shearing to brittle shearing after metallogenesis, which not only led to the activation and migration of copper but also the enrichment of copper in the narrow brittle fractures. Therefore, the ore bodies are characterized by obvious tectonic control.

\section{Mineralization of the Tuwu Copper Deposit}

The Tuwu deposit is located approximately $10 \mathrm{~km}$ north of the Kangguer fault. The southern part of the Tuwu mining area experienced complicated subduction, island arc growth, plate collision, and ductile shearing from the Carboniferous to Permian. Finally, a faulted structural zone with complex structures and properties developed (Han et al., 2006; Wang et al., 2006; Cai et al., 2012), and the obviously superimposed mineralization characteristics of the Tuwu copper deposit developed. By combining and integrating our results with previously published data on the eastern Tianshan orogens, we suggest the following new model to interpret the evolution of the Tuwu deposit in eastern Tianshan and then provide a short discussion. Stage I: In the early Carboniferous, the northward subduction of the North Tianshan Ocean formed the CQ group volcanic rocks in the southern belt of the Dananhu-Haerlik island arc (Hou et al., 2005, 2006). A series of circular structures and radial structures around the volcanic crater were formed in the Tuwu mining area. The intersections of these structures formed dilational zones that provided the space and initial conditions for the emplacement of the plagiogranite porphyry. Typical minerals in the weakly propylitized zone, such as chlorite, epidote, dolomite, and calcite in the CQ Group volcanic rocks, were formed by volcanic hydrothermal processes. The higher contents of trace elements in pyrite and chlorite show that the mixing action of meteoric water played only a relatively minor role, while the high copper contents reflect the high copper background values of the volcanic rocks and the initial enrichment of $\mathrm{Cu}$ by volcanic hydrothermal processes. The volcanic rocks that were far from the ore bodies mainly suffered weakly volcanic hydrothermal process because of the undeveloped fracture system, and the trace element content of chlorite does not change obviously with the spatial position and generally inherited the REE distribution pattern of dark minerals in volcanic rocks Table 3 .

Stage II: In the early Carboniferous, with the subduction and partial melting of the northern Tianshan oceanic plate at depth under certain physical and chemical conditions, adakitic magmas intruded into the shallow crust and formed the plagiogranite porphyry (Wang et al., 2014, Han et al., 2014; Shen et al., 2014b). The contemporaneous faults of the CQ group provided the tectonic environment for porphyry intrusion. The pyrite that formed in the early stage of porphyry mineralization under high-temperature and high-salinity 
conditions has an obvious magmatic-hydrothermal origin. With the development of mineralization, the input of meteoric water, and the long-term metasomatism of fluid in the late stage, the $\mathrm{Co} / \mathrm{Ni}$ ratio of pyrite continuously decreased. From the early to late stages of porphyry mineralization, the REEs in chlorite show similar distribution patterns, indicating that the fluid had a similar source of ore-forming material Table 3. With the addition of meteoric water in the late stage II, athe metallogenic system became more open, and the fluid mixing caused minerogenetic matter precipitation (Liu et al., 2009; Shen et al., 2014b; Wang et al., 2015; Gao et al., 2017; Wang et al., 2018). As a consequence, the REE composition of the fluid changed, and the metallogenic system became a relatively oxidizing environment.

Stage III: In the Late Carboniferous-Triassic, the North Tianshan oceanic basin subducted and closed along the Kangguer ductile shear zone (Xiao et al., 2004; Mao et al., 2014b; Chen et al., 2019). The Tuwu copper deposit, which underwent multiple phases of compression and shear deformation, is located in the influence zone of the Kangguer ductile deformation zone, and the ore body underwent a strong superimposed transformation. The ore body was obviously mylonitized, and the $\mathrm{Co} / \mathrm{Ni}$ ratio of pyrite decreased continuously under the action of multiphase fluids. The trace element characteristics of Chl-III also show distinct differences from those of Chl-II in the porphyry metallogenic stage Table 3.

\section{CONCLUSION}

1) We redefined the altered zones and the following three mineralization stages: premineralization stage (stage I), porphyry metallogenic stage (stage II), and superimposed transformation stage (stage III). Pyrite and chlorite are widely distributed in the three mineralization stages and different altered zones. Elements in pyrite, such as Sb, Se, $\mathrm{Co}$, and $\mathrm{Ni}$, mainly occur in the pyrite crystal lattice via isomorphism. $\mathrm{Cu}$ has obvious positive correlations with $\mathrm{Pb}$, $\mathrm{Zn}, \mathrm{Ag}$, and $\mathrm{Au}$ in different ore-forming stages, and these elements may occur in the form of microinclusions or nanometer-scale particles.

2) The trace element compositions of pyrites from three stages obviously changed, thus reflecting the evolution of fluid properties with the mineralization process. The $\mathrm{Co} / \mathrm{Ni}$ ratios changed from Py-I (11.37-60.60) to Py- $\mathrm{II}_{1}$ (0.09-9.94), Py- $\mathrm{II}_{2}$ (0.12-2.02), and finally Py-III (0.003-0.57), indicating that the fluid evolved from a volcanic genesis to a magmatic hydrothermal genesis. The fluid was affected by meteoric water and other fluids in the late metallogenic period and experienced a superimposed complicated structural hydrothermal fluid.

3) The REE distribution pattern of chlorite has obviously different features in the three stages. Chl-I generally inherited the REE distribution pattern of the volcanic rocks in the CQ Group, which shows that the fluid exsolved from the products of late volcanic activity. The similar REE distribution pattern of $\mathrm{Chl}-\mathrm{II}_{1}$ to $\mathrm{Chl}-\mathrm{II}_{3}$ indicates that the fluid had a similar ore-forming material source. The different features from Chl- $\mathrm{II}_{1}$ to $\mathrm{Chl}-\mathrm{II}_{3}$ show that the fluid evolved from magmatic hydrothermal to the mixture of magmatic and meteoric fluid. The REE distribution pattern of Chl-III shows that the fluid in ductile deformation zones experienced strong water-rock interactions with the surrounding rocks.

4) The contents of $\mathrm{Ti}, \mathrm{V}$, and $\mathrm{Mg}$ in the chlorite decreased exponentially with increasing distance, whereas the contents of $\mathrm{K}, \mathrm{Li}, \mathrm{Ca}, \mathrm{Sr}, \mathrm{Ba}, \mathrm{Mn}, \mathrm{Co}, \mathrm{Ni}, \mathrm{Zn}$, and $\mathrm{Pb}$ increased. The maximum value appears at approximately $1.2 \mathrm{~km}$ and the values then gradually stabilize.

5) The Tuwu porphyry deposit has undergone volcanic activity, porphyry metallogenesis, and structural deformation from the early Carboniferous to early Triassic.

\section{DATA AVAILABILITY STATEMENT}

The raw data supporting the conclusion of this article will be made available by the authors, without undue reservation.

\section{AUTHOR CONTRIBUTIONS}

We the undersigned declare that this manuscript entitled "Mineralization of the Tuwu porphyry $\mathrm{Cu}$ deposit in eastern Tianshan, NW China: Insights from in situ trace elements of chlorite and pyrite" is original, has not been published before, and is not currently being considered for publication elsewhere. We would like to draw the attention of the editor to the following publications of one or more of us that refer to aspects of the manuscript presently being submitted, where relevant copies of such publications are attached. We confirm that the manuscript has been read and approved by all named authors and that there are no other persons who satisfied the criteria for authorship but are not listed. We further confirm that the order of authors listed in the manuscript has been approved by all of us. We understand that the corresponding author is the sole contact for the editorial process. He is responsible for communicating with the other authors about the progress, submissions of revisions, and final approval of proofs. Signed by all authors as follows: WT, QM*, MY, YS, and XL.

\section{FUNDING}

This study was financially supported by the National Key R\&D Program of China (No. 2017YFC0601201), Chinese National Basic Research 973 Program (No. 2014CB440803), Ministry of Land and Resources of China (No. 201411026), and the Chinese Geological Survey Project (No. DD20160071).

\section{ACKNOWLEDGMENTS}

Han Runsheng of the Kunming University of Science and Technology and Researcher Chen Zhengle of the Institute of Geomechanics provided valuable revision suggestions that improved this paper. 


\section{REFERENCES}

Bau, M. (1991). Rare-earth Element Mobility during Hydrothermal and Metamorphic Fluid-Rock Interaction and the Significance of the Oxidation State of Europium. Chem. Geology. 93, 219-230. doi:10.1016/0009-2541(91) 90115-8

Bajwah, Z. U., Seccombe, P. K., and Offler, R. (1987). Trace element distribution, Co: Ni ratios and genesis of the Big Cadia iron-copper deposit, New South Wales, Australia. Mineralium Deposita 22, 292-300. doi:10.1007/BF00204522

Bortnikov, N. S., Gorelikova, N. V., Korostelev, P. G., and Gonevchuk, V. G. (2008). Rare Earth Elements in Tourmaline and Chlorite from Tin-Bearing Assemblages: Factors Controlling Fractionation of REE in Hydrothermal Systems. Geol. Ore Deposits. 50, 445-461. doi:10.1134/s1075701508060032

Bralia, A., Sabatini, G., and Troja, F. (1979). A Revaluation of the Co/Ni Ratio in Pyrite as Geochemical Tool in Ore Genesis Problems. Mineralium Deposita. 14, 353-374. doi:10.1007/bf00206365

Brill, B. (1989). Trace-element Contents and Partitioning of Elements in Ore Minerals from the CSA Cu-Pb-Zn deposit, Australia. Can. Mineral. 27, 263-274. doi:10.1007/BF01164492

Cai, Z. H., Xu, Z. Q., He, B. Z., and Wang, R. R. (2012). Age and Tectonic Evolution of Ductile Shear Zones in the Eastern Tianshan-Beishan Orogenic belt. Acta Petrologica Sinica. 28, 1875-1895. [in Chinese with English abstract]

Campbell, F. A., and Ethier, V. G. (1984). Nickel and Cobalt in Pyrrhotite and Pyrite from the Faro and Sullivan Orebodies. Can. Mineral. 22, 503-506. doi:10. 1007/BF01082965

Chao, F. G., Tu, Q. J., Zhang, X. M., Ren, Y., Li, G. L., and Dong, F. R. (2006). Preliminary Determination of the Early Paleozoic Magmatic Arc in the Karlik Mountains, East Tianshan, Xinjiang, China-Evidence from Zircon SHRIMP $\mathrm{U}-\mathrm{Pb}$ Dating of Granite Bodies in the Tashuihe Area. Geol. Bull. China. 25, 923-927. doi:10.3969/j.issn.1671-2552.2006.08.004 [in Chinese with English abstract]

Chen, F. W., Li, H. Q., Chen, Y. C., Wang, D. H., Wang, J. L., Liu, D. Q., et al. (2005). Zircon SHRIMP U-Pb Dating and its Geological Significance of Mineralizationin Tuwu- Yandong Porphyry Copper Mine, East Tianshan Mountain. Acta Geol. Sinica. 79, 256-261. doi:10.3321/j.issn:0001-5717.2005. 02.011 [in Chinese with English abstract]

Chen, X., Shu, L., Santosh, M., and Zhao, X. (2013). Island Arc-type Bimodal Magmatism in the Eastern Tianshan Belt, Northwest China: Geochemistry, Zircon U-Pb Geochronology and Implications for the Paleozoic Crustal Evolution in Central Asia. Lithos. 168-169, 48-66. doi:10.1016/j.lithos.2012. 10.006

Chen, Y.-J., Chen, H.-Y., Zaw, K., Pirajno, F., and Zhang, Z.-J. (2007). Geodynamic Settings and Tectonic Model of Skarn Gold Deposits in China: An Overview. Ore Geology. Rev. 31, 139-169. doi:10.1016/j. oregeorev.2005.01.001

Chen, Y. J., and Fu, S. G. (1991). Variation of REE Patterns in Early Pre-cambrian Sediments: the Oretical Study and Evidence from the Southern Margin of the Northern China Craton. Chin. Sci. Bull. 36, 1100-1104. doi:10.1159/000202829 [in Chinese with English abstract]

Chen, Y. J., and Zhao, Y. C. (1997). Geochemical Characteristics and Evolution of REE in the Early Precambrian Sediments: Evidences from the Southern Margin of the North China Craton. Episodes. 20, 109-116.

Chen, Z., Xiao, W., Windley, B. F., Schulmann, K., Mao, Q., Zhang, Z., et al. (2019). Composition, Provenance, and Tectonic Setting of the Southern Kangurtag Accretionary Complex in the Eastern Tianshan, NW China: Implications for the Late Paleozoic Evolution of the North Tianshan Ocean. Tectonics. 38, 2779-2802. doi:10.1029/2018tc005385

Cook, N. J., Ciobanu, C. L., Pring, A., Skinner, W., Shimizu, M., Danyushevsky, L., et al. (2009). Trace and Minor Elements in Sphalerite: A LA-ICPMS Study. Geochimica Et Cosmochimica Acta. 73, 4761-4791. doi:10.1016/j.gca.2009. 05.045

Cook, N. J. (1996). Mineralogy of the Sulphide Deposits at Sulitjelma, Northern Norway. Ore Geology. Rev. 11, 303-338. doi:10.1016/s01691368(96)00009-1

Cooke, D. R., Baker, M., Hollings, P., Sweet, G., Chang, Z., Danyushevsky, L., et al. (2014). "New Advances in Detecting the Distal Geochemical Footprints of Porphyry Systems: Epidote mineral Chemistry as a Tool for Vectoring and
Fertility Assessments,". Building Exploration Capability for the 21st Century. Editors KD Kelley and HC Golden (Colorado, USA: Society of Economic Geologists, Special Publication), 18, 127-152

Craig, J. R., Vokes, F. M., and Solberg, T. N. (1998). Pyrite: Physical and Chemical Textures. Mineralium Deposita. 34, 82-101. doi:10.1007/s001260050187

David, R. C., Mike, B., Pete, H., Gabe, S., Zhao, S. G., Zhou, T. F., et al. (2016). New Advances in Detecting the Distal Geochemical Footprints of Porphyry Systems Epidote Mineral Chemistry as a Tool for Vectoring and Fertility Assessments. Society of Economic Geology 18, 127-152. doi:10.5382/SP.18.07

Davidson, J., Tepley, F., Palacz, Z., and Meffan-Main, S. (2001). Magma Recharge, Contamination and Residence Times Revealed by In Situ Laser Ablation Isotopic Analysis of Feldspar in Volcanic Rocks. Earth Planet. Sci. Lett. 184, 427-442. doi:10.1016/s0012-821x(00)00333-2

Deng, X.-H., Wang, J.-B., Pirajno, F., Wang, Y.-W., Li, Y.-C., Li, C., et al. (2016), Re-Os Dating of Chalcopyrite from Selected mineral Deposits in the Kalatag District in the Eastern Tianshan Orogen, China. Ore Geology. Rev. 77, 72-81. doi:10.1016/j.oregeorev.2016.01.014

Fadda, S., Fiori, M., Grillo, S. M., and Prochaska, W. (2012). REE Mobilization in Complex Hydrothermal-Metasomatic Systems: Fluid Chemistry Evidence of Albitite and Chlorite-Talc Mineralisations in Central Sardinia, Italy. Proc. 10th Int. Congress Appl. Mineralogy, 171-178. doi:10.1007/978-3-642-27682-8_22

Fang, T. H., Qin, K. Z., Wang, S. L., Wang, S. L., Jiang, F. Z., Gan, X. P., and Zhou, Z. J. (2002). Preliminary analysis on the geological back ground of Kalatage $\mathrm{Cu}-$ Au deposit, Xinjiang. Mineral Deposits 21, 380-384. [in Chinese with English abstract]

Foster, M. D. (1962). Interpretation of the Composition and a Classification of the Chlorites. U.s.geological Surv. Prof. Paper. 414, 33. doi:10.3133/pp414A

Franchini, M., Mcfarlane, C., Maydagán, L., Reich, M., Lentz, D. R., Meinert, L., et al. (2015). Trace Metals in Pyrite and Marcasite from the Agua Rica Porphyry-High Sulfidation Epithermal deposit, Catamarca, Argentina: Textural Features and Metal Zoning at the Porphyry to Epithermal Transition. Ore Geology. Rev. 66, 366-387. doi:10.1016/j.oregeorev.2014.10.022

Fulignati, P., Gioncada, A., and Sbrana, A. (1999). Rare-earth Element (REE) Behaviour in the Alteration Facies of the Active Magmatic-Hydrothermal System of Vulcano (Aeolian Islands, Italy). J. Volcanology Geothermal Res. 88, 325-342. doi:10.1016/s0377-0273(98)00117-6

Gao, J.-F., Zhou, M.-F., Qi, L., Chen, W. T., and Huang, X.-W. (2015). Chalcophile Elemental Compositions and Origin of the Tuwu Porphyry Cu deposit, NW China. Ore Geology. Rev. 66, 403-421. doi:10.1016/j.oregeorev.2014.08.009

Gao, J. F., Zhou, M. F., Zhang, L. C., Chen, W. T., and Huang, X. W. (2017). Chalcophile elemental compositions and origin of the Tuwu porphyry Cudeposit, NW China. Ore Geology Reviews 66, 403-421. doi:10.1016/j. oregeorev.2014.08.009

Gao, J. G., Li, W. Y., Guo, X. C., Zhou, Y., and Fan, T. B. (2014). Geochemistry, Zircon U-Pb Age and Hf Isotopes of Late Carboniferous Rift Volcanics in the Sepikou Region, Eastern Bogda, Xinjiang. Acta Petrologica Sinica. 30, 3539-3552. [in Chinese with English abstract]

Gu, L. X., Hu, S. X., Yu, C. S., Wu, C. Z., and Yan, Z. (2001a). Initiation and Evolution of the Bogda Subduction-torn-type Rift. Acta Petrologica Sinica. 17, 585-597. doi:10.3321/j.issn:1000-0569.2001.04.009 [in Chinese with English abstract]

Gu, L. X., Hu, S. X., Yu, C. S., Zhao, M., Wu, C. Z., and Li, H. Y. (2001b). Intrusive Activities during Compression-Extension Tectonic Conversion in the Bogda Intracontinental Orogen. Acta Petrologica Sinica. 2, 187-198. doi:10.3321/j.issn: 1000-0569.2001.02.002 [in Chinese with English abstract]

Guo, H. C., Zhong, L., and Li, L. Q. (2006). Zircon SHRIMP U-Pb Dating of Quartz Diorite in the Koumenzi Area, Karlik Mountains, East Tianshan, Xinjiang, China, and its Geological Significance. Geol. Bull. China 25, 928-931. doi:10. 3969/j.issn.1671-2552.2006.08.005 [in Chinese with English abstract]

Han, B. F., Song, B., Chen, L. H., and Li, Z. H. (2004). Zircon U-Pb SHRIMP Ages of the Mafic-Ultramafic Complexes from the Halatongke and Huangshandong $\mathrm{Cu}-\mathrm{Ni}$ Sulfide Deposits in Xinjiang, and Their Tectonic Significance. Chin. Sci. Bull. 49, 2324-2328. [in Chinese with English abstract]. doi:10.1360/04wd0163

Han, C. M., Xiao, W. J., Zhao, G. C., Mao, J. W., and Mao, Q. G. (2004). Major Types, Characteristics and Geodynamic Mechanism of Upper Paleozoic Copper Deposits in Northern Xinjiang, Northwestern China. Ore Geology. Rev. 28, 308-328. doi:10.1016/j.oregeorev.2005.04.002 
Han, C. M., Xiao, W. J., Wan, B., Ao, S. J., Zhang, J. E., Song, D. F., et al. (2018). Late Palaeozoic-Mesozoic Endogenetic Metallogenic Series and Geodynamic Evolution in the East Tianshan Mountains. Acta Petrologica Sinica. 34, 1914-1932. [in Chinese with English abstract]

Han, C., Xiao, W., Zhao, G., Ao, S., Zhang, J., Qu, W., et al. (2010). In-situ U-Pb, Hf and $\mathrm{Re}$-os Isotopic Analyses of the Xiangshan $\mathrm{Ni}-\mathrm{Cu}-\mathrm{Co}$ deposit in Eastern Tianshan (Xinjiang), Central Asia Orogenic Belt: Constraints on the Timing and Genesis of the Mineralization. Lithos. 120, 547-562. doi:10.1016/j.lithos. 2010.09.019

Han, C., Xiao, W., Zhao, G., Mao, J., Yang, J., Wang, Z., et al. (2006). Geological Characteristics and Genesis of the Tuwu Porphyry Copper deposit, Hami, Xinjiang, Central Asia. Ore Geology. Rev. 29, 77-94. doi:10.1016/j.oregeorev.2005.07.032

Han, Z. K., Han, C. M., Xiao, W. J., Zhao, G. C., Wang, Z. M, Ao, S. J., et al. (2014). Palaeozoic porphyry $\mathrm{Cu}-\mathrm{Au}$ and ultramafic $\mathrm{Cu}-\mathrm{Ni}$ deposits in the eastern Tianshan orogenic belt: Temporal constraints from U-Pb geochronology. Inter. Geol. Review 55, 842-862. doi:10.1080/00206814.2012.745977

Hedenquist, J. W. (1995). "The Ascent of Magmatic Fluid, Discharge versus Mineralization,". Magmas, Fluids and Ore Deposits Mineralogical Association of Canada, Short Course. Editor JFH Thompson, 23, 263-289.

Hou, G. S., Tang, H. F., and Liu, C. Q. (2006). Geochemical Characteristics of the Late Paleozoic Volcanics in Jueluotage Tectonic belt, Eastern Tianshan and its Implications. Acta Petrologica Sinica. 22, 1167-1177. doi:10.3321/j.issn:10000569.2006.05.009 [in Chinese with English abstract]

Hou, G. S., Tang, H. F., Liu, C. Q., and Wang, Y. B. (2005). Geochronological and Geochemical Study on the Wallrock of Tuwu-Yandong Porphyry Copper Deposits, Eastern Tianshan Mountains. Acta Petrologica Sinica. 21, 1729-1736. doi:10.3969/j.issn.1000-0569.2005.06.020 [in Chinese with English abstract]

Inoue, A., Kurokawa, K., and Hatta, T. (2010). Application of Chlorite Geothermometry to Hydrothermal Alteration in Toyoha Geothermal System, Southwestern Hokkaido, Japan. Resource Geology. 60, 52-70. doi:10. 1111/j.1751-3928.2010.00114.x

Karakaya, M. Ç., Karakaya, N., Küpeli, Ş., and Yavuz, F. (2012). Mineralogy and Geochemical Behavior of Trace Elements of Hydrothermal Alteration Types in the Volcanogenic Massive Sulfide Deposits, NE Turkey. Ore Geology. Rev. 48, 197-224. doi:10.1016/j.oregeorev.2012.03.007

Keith, M., Häckel, F., Haase, K. M., Schwarz-Schampera, U., and Klemd, R. (2016). Trace Element Systematics of Pyrite from Submarine Hydrothermal Vents. Ore Geology. Rev. 72, 728-745. doi:10.1016/j.oregeorev.2015.07.012

Khalili, M., and Malekmahmoudi, F. (2012). "Geochemical Variations during Alteration of an Andesite-Basalt to Bentonite, in Khur, East of Isfahan, Iran," in Proceedings of the 10th International Congress for Applied Mineralogy (ICAM), 43, 361-368. doi:10.1007/978-3-642-27682-8_43

Large, R. R., Bull, S. W., and Maslennikov, V. V. (2011). A Carbonaceous Sedimentary Source-Rock Model for Carlin-Type and Orogenic Gold Deposits. Econ. Geology. 106, 331-358. doi:10.2113/econgeo.106.3.331

Large, R. R., Danyushevsky, L., Hollit, C., Maslennikov, V., Meffre, S., Gilbert, S., et al. (2009). Gold and Trace Element Zonation in Pyrite Using a Laser Imaging Technique: Implications for the Timing of Gold in Orogenic and Carlin-Style Sediment-Hosted Deposits. Econ. Geology. 104, 635-668. doi:10.2113/ gsecongeo.104.5.635

Large, R. R., Maslennikov, V. V., Robert, F., Danyushevsky, L. V., and Chang, Z. (2007). Multistage Sedimentary and Metamorphic Origin of Pyrite and Gold in the Giant Sukhoi Log Deposit, Lena Gold Province, Russia. Econ. Geology. 102, 1233-1267. doi:10.2113/gsecongeo.102.7.1233

Leng, C. B. (2017). Genesis of Hongshan Cu Polymetallic Large deposit in the Zhongdian Area, NW Yunnan, Constraints from LA-IPC-MS of Trace Elements of Pyrite and Pyrrhotite. Earth Sci. Front. 24, 162-175. doi:10. 13745/j.esf.yx.2016-11-61 [in Chinese with English abstract]

Li, H. Q., Chen, F. W., Lu, Y. F., Yang, H. M., Guo, J., and Mei, Y. P. (2004). Zircon SHRIMP U-Pb Age and Strontium Isotopes of Mineralized Granitoids in the Sanchakou Copper Polymetallic Depoist, East Tianshan Mountains. Acta Geologica Sinica. 22, 191-195. doi:10.3321/j.issn:1006-3021.2004.02.018 [in Chinese with English abstract]

Li, J. Y., Song, B., Wang, K. Z., Li, Y. P., Sun, G. H., and Qi, D. Y. (2006). Permian mafic-ultramafic complexes on the southern margin of the Tu-Ha basin, eastern Tianshan Mountians: geological records of vertical crustal growth in central
Asia. Acta. Geoscientica Sinica 27, 424-446. doi:10.3321/j.issn:1006-3021.2006. 05.006 [in Chinese with English abstract]

Li, F. M., Wang, Z. S., and Hou, W. B. (2002). Synthetic prospectiong model developed from Xiaorequanzi copper deposit in east Tianshan, Xinjiang. Xingjiang Geology 20, 38-43. doi:10.3969/j.issn.1000-8845.2002.01.009 [in Chinese with English abstract]

Li, W., Chen, J. L., Dong, Y. P., Xu, X. Y., Li, Z. P., Liu, X. M., et al. (2016). Early Paleozoic subduction of the Paleo-Asian Ocean: Zircon U-Pb geochronological and geochemical evidence from the Kalatag high-Mg andesites, East Tianshan. Acta Petrologica. Sinica. 32, 505-521. [in Chinese with English abstract]

Liu, M., Wang, Z. L., Zhang, Z. H., Chen, W. S., and Yang, D. (2009). Fluid Inclusion Geochemistry of Tuwu Porphyry Copper deposit, Eastern Tianshan in Xinjiang. Acta Geologica Sinica. 25, 1446-1455. [in Chinese with English abstract]

Long, L. L., Wang, J. B., Wang, Y., Deng, X. H., Mao, Q. G., Sun, Y., et al. (2019). Metallogenic Regularity and Metallogenic Model of the Paleo Arc-basin System in Eastern Tianshan. Acta Petrologica Sinica. 35, 3161-3188. [in Chinese with English abstract]

Long, L. L., Wang, J. B., Wang, Y. W., Mao, Q. G., Deng, X. H., Zhao, L. T., et al. (2016). Discussion on the Age of Ore-Hosting Volcanic Strata in Kalatage Ore Concentration Area, Eastern Tianshan, Xinjiang. Mineral. Exploration. 7, 31-37. doi:10.3969/j.issn.1674-7801.2016.01.004 [in Chinese with English abstract]

Lowell, J. D., and Guilbert, J. M. (1970). Lateral and Vertical AlterationMineralization Zoning in Porphyry Ore Deposits. Econ. Geology. 65, 373-408. doi:10.2113/gsecongeo.65.4.373

Ma, R. S., Shu, L. S., and Sun, J. (1997). Tectonic Evolution and Metalogeny of Eastern Tianshan Mountains. Beijing: Geological Publishing House

Ma, T. L., Wang, L. Q., Sun, L. Q., and Shu, B. (2003). Application of Magnetic Fabric Analysis to the Ductile Deformation Belt. Acta Geoscientia Sinica. 449-452. doi:10.3321/j.issn:1006-3021.2003.05.010 [in Chinese with English abstract]

MacLean, W. H. (1988). Rare Earth Element Mobility at Constant Inter-REE Ratios in the Alteration Zone at the Phelps Dodge Massive Sulphide deposit, Matagami, Quebec. Mineral. Deposita. 23, 231-238. doi:10.1007/ bf00206399

Mao, J. W., Yang, J. M., Qu, W. J., Du, A. D., Wang, Z. L., and Han, C. M. (2002). Re-Os Dating of Cu-Ni Sulfide Ores from Huangshandong deposit in Xinjiang and its Geodynamic Significance. Mineral. Deposits. 21, 323-330. doi:10.1007/ s11769-002-0037-5

Mao, Q. G., Fang, T. H., Wang, J. B., Wang, S. L., and Wang, N. (2010). The Geochronology Studies of the Early Paleozoic Honghai Massive Sulfide Deposits and its Geological Significance Kalatage belt, in Eastern Tianshan Mountain, Xinjiang, Northwest China. Acta Petrologica Sinica. 26, 3017-3026. [in Chinese with English abstract]

Mao, Q. G., Wang, J. B., Fang, T. H., Yu, M. J., and Sun, Y. (2017). Discovery of the Middle Devonian Yudai Porphyric Cu(Au) Deposit in the Kalatage Area of Eastern Tianshan Mountain,Xinjiang and and its Geological Prospecting Significance. Geology Exploration 53, 1-11. [in Chinese with English abstract]

Mao, Q. G., Wang, J. B., Fang, T. H., Yu, M. J., Zhu, J. J., Zhuang, R., et al. (2016). Geological Characteristics of Honghai VMS Type deposit of Kalatage Ore belt in the Eastern Tianshan. Mineral. Exploration. 7, 17-30. [in Chinese with English abstract]

Mao, Q. G., Wang, J. B., Yu, M. J., Ao, S. J., and Li, Y. C. (2020). Re-Os and U-Pb Geochronology for the Xiaorequanzi VMS deposit in the Eastern Tianshan, NW China: Constraints on the Timing of Mineralization and Stratigraphy. Ore Geology. Rev. 122, 1-14. doi:10.1016/j.oregeorev.2020.103473

Mao, Q. G., Xiao, W. J., Han, C. M., Sun, M., Yuan, C., Yan, Z., et al. (2006). Zirocn $\mathrm{U}-\mathrm{Pb}$ Age and the Geochemistry of the Baishiquan Mafic-Ultramafic Complex in the Eastern Tianshan, Xinjiang: Constraints on the Closure of the PaleoAsian Ocean. Acta Petrologica Sinica 22, 153-162. doi:10.3321/j.issn:1000-0569. 2006.01.016 [in Chinese with English abstract]

Mao, Q. G., Xiao, W. J., Windley, B. F., yU, M. J., and Zhang, J. E. (2019). Early Permian Subduction-Related Transtension in the Turpan Basin, East Tianshan (NW China): Implications for Accretionary Tectonics of the Southern Altaids. Geol. Mag., 1-24. 
Mao, Q., Wang, J., Xiao, W., Fang, T., Yu, M., Ao, S., et al. (2014a). Stratigraphic, $\mathrm{U}-\mathrm{Pb}$ (Zircon) and Geochemical Constraints on Magmas, Mineralization and Geological Evolution of the Kalatage District, the Central Part of Dananhu Arc in Eastern Tianshan Mountains. Acta Geologica Sinica - English Edition. 88, 885-886. [in Chinese with English abstract]. doi:10.1111/1755-6724. 12376_11

Mao, Q., Xiao, W., Fang, T., Windley, B. F., Sun, M., Ao, S., et al. (2014b). Geochronology, Geochemistry and Petrogenesis of Early Permian Alkaline Magmatism in the Eastern Tianshan: Implications for Tectonics of the Southern Altaids. Lithos. 190-191, 37-51. doi:10.1016/j.lithos.2013.11.011

Mao, Q., Yu, M., Xiao, W., Windley, B. F., Li, Y., Wei, X., et al. (2018). Skarnmineralized Porphyry Adakites in the Harlik Arc at Kalatage, E. Tianshan (NW China): Slab Melting in the Devonian-Early Carboniferous in the Southern Central Asian Orogenic Belt. J. Asian Earth Sci. 153, 365-378. doi:10.1016/j. jseaes.2017.03.021

Michard, A., and Albarède, F. (1986). The REE Content of Some Hydrothermal Fluids. Chem. Geology. 55, 51-60. doi:10.1016/0009-2541(86)90127-0

Pan, H. D., Shen, P., Chen, G., Yang, J. T., and Dai, H. W. (2013). Volcanic-plutonic Complex, Ore-Forming Rocks and Their Alterations in Tuwu Porphyry $\mathrm{Cu}$ deposit of Xinjiang. Mineral. Deposits. 32, 794-808.

Parsapoor, A., Khalili, M., and Mackizadeh, M. A. (2009). The Behaviour of Trace and Rare Earth Elements (REE) during Hydrothermal Alteration in the Rangan Area (Central Iran). J. Asian Earth Sci. 34, 123-134. doi:10.1016/j.jseaes.2008. 04.005

Pirajno, F. (2009). Hydrothermal Processes and Mineral System. Berlin, Germany: Springer Netherlands

Pirajno, F. (2013). The Geology and Tectonic Settings of China's Mineral Deposits. Berlin, Germany: Springer Netherlands. doi:10.1007/978-94-007-4444-8

Qin, K.-z., Su, B.-x., Sakyi, P. A., Tang, D.-m., Li, X.-h., Sun, H., et al. (2011). SIMS Zircon U-Pb Geochronology and Sr-Nd Isotopes of Ni-Cu-Bearing MaficUltramafic Intrusions in Eastern Tianshan and Beishan in Correlation with Flood Basalts in Tarim Basin (NW China): Constraints on a Ca. 280 Ma Mantle Plume. Am. J. Sci. 311, 237-260. doi:10.2475/03.2011.03

Qin, Y. S., Zhang, J. C., Ding, K. S., Xu, Y. X., Tang, D. M., Xu, X. W., et al. (2009). Semi-empirical Likelihood Confidence Intervals for the Differences of Quantiles with Missing Data. Acta Math. Sin.-English Ser. 25, 845-854. [in Chinese with English abstract]. doi:10.1007/s10114-009-6476-5

Reed, M. H. (1997). "Hydrothermal Alteration and its Relationship to Ore Fluid Composition," in Geochemistry of Hydrothermal Ore Deposits. Editor H. L. Barnes. third ed. (New York: Wiley), 1-29.

Reich, M., Deditius, A., Chryssoulis, S., Li, J.-W., Ma, C.-Q., Parada, M. A., et al. (2013). Pyrite as a Record of Hydrothermal Fluid Evolution in a Porphyry Copper System: A SIMS/EMPA Trace Element Study. Geochimica Et Cosmochimica Acta. 104, 42-62. doi:10.1016/j.gca.2012.11.006

Rui, Z. Y., Wang, F. T., Li, H. H., Dong, L. H., Wang, L., Jiang, L. F., et al. (2001). New Progress in the Eastern Tianshan Porphyry Copper belt. Chin. Geology. 28, 11-16. doi:10.3969/j.issn.1000-3657.2001.02.003 [in Chinese with English abstract]

Rui, Z. Y., Wang, L. S., and Wang, Y. T. (2002). Discussion on Metallogenic Epoch of Tuwu and Yandong Porphyry Copper Deposits in Eastern Tianshan Mountains, Xinjiang. Mineral. Deposits. 21, 16-22. doi:10.3969/j.issn.02587106.2002.01.003 [in Chinese with English abstract]

Şengör, A. M. C., Natal'in, B. A., and Burtman, U. S. (1993). Evolution of the Altaid tectonic collage and Paleozoic crustal growth in Eurasia. Nature 364, 209-304. doi:10.1038/364299a0

Shen, P., Pan, H. D., Dong, L. H., Yang, J. T., Shen, Y. C., Dai, H. W., et al. (2012). Caldera Complex, Hosted Rocks and Alteration of the Yandong Porphyry Copper deposit in Eastern Tianshan, Xinjiang. Acta Petrologica Sinica. 28, 1966-1980. [in Chinese with English abstract]

Shen, P., Pan, H., and Dong, L. (2014a). Yandong Porphyry Cu deposit, Xinjiang, China-geology, Geochemistry and Sims U-Pb Zircon Geochronology of Host Porphyries and Associated Alteration and Mineralization. J. Asian Earth Sci. 80, 197-217. doi:10.1016/j.jseaes.2013.11.006

Shen, P., Pan, H., Zhou, T., and Wang, J. (2014b). Petrography, Geochemistry and Geochronology of the Host Porphyries and Associated Alteration at the Tuwu $\mathrm{Cu}$ deposit, NW China: a Case for Increased Depositional Efficiency by Reaction with Mafic Hostrock? Miner Deposita. 49, 709-731. doi:10.1007/ s00126-014-0517-4
Shu, L., Wang, B., Zhu, W., Guo, Z., Charvet, J., and Zhang, Y. (2011). Timing of Initiation of Extension in the Tianshan, Based on Structural, Geochemical and Geochronological Analyses of Bimodal Volcanism and Olistostrome in the Bogda Shan (NW China). Int. J. Earth Sci. (Geol Rundsch). 100, 1647-1663. doi:10.1007/s00531-010-0575-5

Song, B., Li, J. Y., Li, W. P., Wang, K. Z., and Wang, Y. B. (2002). SHRIMP Dating of the Dananhu and Kezirkalasayi Granitoids Batholiths in Southern Margin of Tuha Basin and Their Geological Implication. Xingjiang Geology. 20, 332-345. [in Chinese with English abstract]

Song, X. X., and Zhang, J. K. (1986). Minor Elements in Pyrite of Various Genetic Types Form China. Bulletin of the Institute of mineral Deposites, Chinese Academy of Geological Sciences. Beijing: Geological Publishing House

Sun, G. H., Li, J. Y., Gao, L. M., and Yang, T. N. (2005). Zircon SHRIMP U-Pb Age of a Dioritic Pluton in the Harlik Mountain, Eastern Xinjiang, and its Tectonic Implication. Geol. Rev. 51, 463-469. doi:10.3321/j.issn:0371-5736.2005.04.015 [in Chinese with English abstract]

Sun, Y., Wang, J. B., Li, Y. C., Wang, Y. W., Yu, M. J., Long, L. L., et al. (2017). Recognition of Late Ordovician Yudai Porphyry Cu (Au, Mo) Mineralization in the Kalatag District, Eastern Tianshan Terrane, NW China: Constraints from Geology, Geochronology, and Petrology. Ore Geology. Rev. 100, 220-236. doi:10.1016/j.oregeorev.2017.07.011

Sverjensky, D. A. (1984). Europium Redox Equilibria in Aqueous Solution. Earth Planet. Sci. Lett. 67, 70-78. doi:10.1016/0012-821x(84)90039-6

Tan, W., Han, R. S., Wang, L., Liu, F., Guo, Y. X. Y., Wang, M. Z., et al. (2016). REE Geochemical of Gold-Polymetallic deposit in Beiya, Western Yunnan Province. J. Chin. Soc. rare earths. 34, 113-128. [in Chinese with English abstract]

Tan, W., Han, R. S., Wang, L., Liu, F., Wang, M. Z., and Guo, Y. X. Y. (2018). Iron Source of the Beiya Porphyry-type Au-Polymetallic deposit in Northwestern Yunnan, China. Geochimica. 47, 541-553. doi:10.19700/j.0379-1726.2018.05. 007 [in Chinese with English abstract].

Tang, J. H., Gu, L. X., Zheng, Y. C., Fang, T. H., Zhang, Z. Z., Gao, J. H., et al. (2006). Petrology, Geochemistry and Genesis of the Na-Rich Volcanic Rocks of the Kalatag Area, Eastern Tianshan. Acta Petrologica Sinica. 22, 1150-1166. doi:10. 3321/j.issn:1000-0569.2006.05.008 [in Chinese with English abstract]

Taylor, S. R., and Mclennan, S. M. (1985). The Continental Crust: Its Composition and Evolution. J. Geology. 94, 57-72. doi:10.1086/629067

Terakado, Y., and Fujitani, T. (1998). Behavior of the Rare Earth Elements and Other Trace Elements during Interactions between Acidic Hydrothermal Solutions and Silicic Volcanic Rocks, Southwestern Japan. Geochimica Et Cosmochimica Acta. 62, 1903-1917. doi:10.1016/s0016-7037(98)00109-4

Wang, F. T., Feng, J., Hu, J. W., Wang, L., Jiang, L. F., and Zhang, Z. (2001). The geological characteristics and discovery significance of Tuwu porphyry copper deposit, Xinjiang. Geology. China 28, 36-39. doi:10.1007/s00126-018-0859-4 [in Chinese with English abstract]

Wang, J. B., Wang, Y. W., and He, Z. J. (2006). Ore Deposits as a Guide to the Tectonic Evolution in the East Tianshan Mountains,NW China. Geology. China, 461-469. [in Chinese with English abstract]

Wang, J. R., Li, T. D., Tian, L. P., Yu, M., Wang, H. T., Zhao, Z. X., et al. (2010). Late Paleozoic Tectono-Magmatic Evolution in Bogda Orogenic Belt, Xinjiang: Evidence from Geochemistry of Volcanic Rocks. Acta Petrologica Sinica 26, 1103-1115. [in Chinese with English abstract]

Wang, K. R. (1989). Genesis Mineralogy of the Earth and Universe. Hefei, Anhui Education Press

Wang, Y.-H., Xue, C.-J., Liu, J.-J., Wang, J.-P., Yang, J.-T., Zhang, F.-F., et al. (2015). Early Carboniferous Adakitic Rocks in the Area of the Tuwu deposit, Eastern Tianshan, NW China: Slab Melting and Implications for Porphyry Copper Mineralization. J. Asian Earth Sci. 103, 332-349. doi:10.1016/j.jseaes. 2014.09.032

Wang, Y., Chen, H., Baker, M. J., Han, J., Xiao, B., Yang, J., et al. (2018). Multiple Mineralization Events of the Paleozoic Tuwu Porphyry Copper deposit, Eastern Tianshan: Evidence from Geology, Fluid Inclusions, Sulfur Isotopes, and Geochronology. Miner Deposita. 54, 1053-1076. doi:10.1007/s00126-018$0859-4$

Wang, Y. F., Chen, H. Y., Xiao, B., Han, J. S., and Yang, J. T. (2016). Porphyriticoverlapped Mineralization of Tuwu and Yandong Copper Deposits in Eastern Tianshan Mountains, Xinjiang. Mineral. Deposits. 35, 51-68. doi:10.16111/j. 0258-7106.2016.01.004 [in Chinese with English abstract] 
Wang, Y. L., Zhang, Z. W., Zhang, J. W., You, M. X., Li, K., and Wang, B. L. (2016). Mineralogy and Sr-Nd Isotope Characteristics of Baixintan Ni-Cu deposit in Eastern Tianshan Mountains, Xinjiang, and Mineralization Process. Acta Geologica Sinica. 90, 2747-2758. [in Chinese with English abstract]

Wang, Y. H., Xue, C. J., Liu, J. J., Wang, J. P., Yang, J. T., Zhang, F. F., et al. (2014). Geochemistry, Geochronology, Hf Isotope, and Geological Significance of the Tuwu Porphyry Copper deposit in Eastern Tianshan, Xinjiang. Acta Petrologica Sinica. 30, 3383-3399. [in Chinese with English abstract]

Wilkinson, J. J., Chang, Z., Cooke, D. R., Baker, M. J., Wilkinson, C. C., Inglis, S., et al. (2015). The Chlorite Proximitor: A New Tool for Detecting Porphyry Ore Deposits. J. Geochemical Exploration. 152, 10-26. doi:10.1016/j.gexplo.2015. 01.005

Wu, H., Li, H. Q., Chen, F. W., Lu, Y. F., Deng, G., Mei, Y. P., et al. (2006). Zircon SHRIMP U-Pb Dating of Plagiogranite Porphyry in the Chihu MolybdenumCopper District, Hami, East Tianshan. Geol. Bull. China 25, 549-552. doi:10. 3969/j.issn.1671-2552.2006.05.003 [in Chinese with English abstract]

Xiao, B., Chen, H., Hollings, P., Wang, Y., Yang, J., and Wang, F. (2018). Element Transport and Enrichment during Propylitic Alteration in Paleozoic Porphyry $\mathrm{Cu}$ Mineralization Systems: Insights from Chlorite Chemistry. Ore Geology. Rev. 102, 437-448. doi:10.1016/j.oregeorev.2018.09.020

Xiao, B., Chen, H. Y., Wang, Y. F., and Yang, J. T. (2017). Zircon U-Pb and Molybdenite Re-os Dating of the Tuwu-Yandong Cu Deposit Belt of the Eastern Tianshan Mountains and its Geological Significance. Geotectonica et Metallogenia 41, 145-156. doi:10.16539/j.ddgzyckx.2016.06.020

Xiao, W.-J., Zhang, L. C., Qin, K. Z., Sun, S., and Li, J. L. (2004). Paleozoic Accretionary and Collisional Tectonics of the Eastern Tianshan (China): Implications for the continental Growth of central Asia. Am. J. Sci. 304, 370-395. doi:10.2475/ajs.304.4.370

Xiao, W., Windley, B. F., Sun, S., Li, J., Huang, B., Han, C., et al. (2015). A Tale of Amalgamation of Three Permo-Triassic Collage Systems in Central Asia: Oroclines, Sutures, and Terminal Accretion. Annu. Rev. Earth Planet. Sci. 43, 477-507. doi:10.1146/annurev-earth-060614-105254

Yang, K., and Scott, S. D. (1996). Possible Contribution of a Metal-Rich Magmatic Fluid to a Sea-Floor Hydrothermal System. Nature. 383, 420-423. doi:10.1038/383420a0

Yao, F. J. (2006). The Study of Spectral Feature and Extraction of Remote Sensing Alteration Anomalies for Metallic Ore Deposits in Gobi Arid Zone and Application in mineral Resources. Master Thesis. Beijing: Chinese Academy of Geological Sciences.

Ye, L., Cook, N. J., Ciobanu, C. L., Yuping, L., Qian, Z., Tiegeng, L., et al. (2011). Trace and Minor Elements in Sphalerite from Base Metal Deposits in South China: A LA-ICPMS Study. Ore Geology. Rev. 39, 188-217. doi:10.1016/j.oregeorev.2011.03.001

Yu, M., Wang, Y., Wang, J., Mao, Q., Deng, X., Sun, Y., et al. (2019). The Mineralization of the Kalatage Arc, Eastern Tianshan, NW China: Insights from the Geochronology of the Meiling $\mathrm{Cu}-\mathrm{Zn}(-\mathrm{Au})$ deposit. Ore Geology. Rev. 107, 72-86. doi:10.1016/j.oregeorev.2018.12.009

Zang, W., and Fyfe, W. S. (1995). Chloritization of the Hydrothermally Altered Bedrock at the Igarapé Bahia Gold deposit, Carajás, Brazil. Mineralium Deposita. 30, 30-38. doi:10.1007/bf00208874
Zhai, Y. S., Yao, S. Z., and Cai, K. Q. (2011). Ore deposit Science. Third edition. Beijing: Geological Publishing House. doi:10.1145/2063348.2063363

Zhang, L., Xiao, W., Qin, K., and Zhang, Q. (2006). The Adakite Connection of the Tuwu-Yandong Copper Porphyry belt, Eastern Tianshan, Nw china: Trace Element and Sr-Nd-Pb Isotope Geochemistry. Miner Deposita. 41, 188-200. doi:10.1007/s00126-006-0058-6

Zhang, R., Mao, Q. G., Yu, M. J., Fang, T. H., and Cheng, F. W. (2017). Geochemical Characteristics and Zircon U-Pb Ages of the Magmatite in the Qiongdukuke Ag-Pb-Zn Polymetallic deposit of Xinjiang. Geology. Exploration. 53, 270-282. [in Chinese with English abstract]

Zhang, W. D., Wu, X. B., Deng, X. H., Mao, Q. G., Zhang, H. Q., Yang, L. Y., et al. (2018). Fluid Inclusions Constraints on the Origin of the Xiaorequanzi Deposit in Eastern Tianshan. Diqiu Kexue Zhongguo Dizhi Daxue Xuebao/earth Sci. J. China Univ. Geosciences. 43, 3036-3048. doi:10.3799/dqkx.2018.150

Zhang, W., Lentz, D. R., Thorne, K. G., and Massawe, R. J. R. (2020). Late SilurianEarly Devonian Slab Break-Off beneath the Canadian Appalachians: Insights from the Nashwaak Granite, West-central New Brunswick, Canada. Lithos. 358-359, 105393. doi:10.1016/j.lithos.2020.105393

Zhao, Z. N., Wang, Y. H., Wang, J. P., Dong, L. S., Wang, H., Zhang, F. F., et al. (2014). Geochemistry and Geochronology of Diorite Porphyrite of the Tuwu Copper Deposit and its Geological Significance, Eastern Tianshan. Acta Mineralogica Sinica. 34, 332-349. doi:10.16461/j.cnki. 1000-4734.2014.04.012 [in Chinese with English abstract]

Zhou, J. Y., Cui, B. F., Xiao, H. L., Chen, S. Z., and Zhu, D. M. (2001). The Kangguertag-Huangshan Collision Zone of Bilateral Subduction and its Metallogenic Model and Programs in Xinjiang, China. Volcanology Mineral. Resour. 22, 252-263.

Zhou, M.-F., Michael Lesher, C., Yang, Z., Li, J., and Sun, M. (2004). Geochemistry and Petrogenesis of $270 \mathrm{Ma} \mathrm{Ni-Cu-(PGE)} \mathrm{Sulfide-Bearing}$ Mafic Intrusions in the Huangshan District, Eastern Xinjiang, Northwest China: Implications for the Tectonic Evolution of the Central Asian Orogenic belt. Chem. Geology. 209, 233-257. doi:10.1016/j.chemgeo. 2004.05.005

Zhou, T. F., Zhang, L. J., Yuan, F., Fan, Y., and David, R. C. (2010). LA-ICP-MS In Situ Trace Element of Pyrite from the Xinqiao $\mathrm{Cu}-\mathrm{Au}-\mathrm{S}$ deposit in Tongling, Anhui, and its Constraints on the Ore Genesis. Earth Sci. Front. 17, 306-319. [in Chinese with English abstract]

Conflict of Interest: WT, QM, MY, YS, and XL were employed by the company Beijing Institute of Geology for Mineral Resources Co., Ltd., and WT and XL were employed by the company Sino-Zijin Resources Ltd.

Copyright (c) 2021 Tan, Mao, Yu, Sun and Lv. This is an open-access article distributed under the terms of the Creative Commons Attribution License (CC $B Y)$. The use, distribution or reproduction in other forums is permitted, provided the original author(s) and the copyright owner(s) are credited and that the original publication in this journal is cited, in accordance with accepted academic practice. No use, distribution or reproduction is permitted which does not comply with these terms. 